\title{
Mirror Chern numbers in the hybrid Wannier representation
}

\author{
Rauch, Tomáš; Olsen, Thomas; Vanderbilt, David; Souza, Ivo
}

Published in:

Physical Review B

Link to article, DOI:

10.1103/PhysRevB.103.195103

Publication date:

2021

Document Version

Publisher's PDF, also known as Version of record

Link back to DTU Orbit

Citation (APA):

Rauch, T., Olsen, T., Vanderbilt, D., \& Souza, I. (2021). Mirror Chern numbers in the hybrid Wannier representation. Physical Review B, 103(19), [195103]. https://doi.org/10.1103/PhysRevB.103.195103

\section{General rights}

Copyright and moral rights for the publications made accessible in the public portal are retained by the authors and/or other copyright owners and it is a condition of accessing publications that users recognise and abide by the legal requirements associated with these rights.

- Users may download and print one copy of any publication from the public portal for the purpose of private study or research.

- You may not further distribute the material or use it for any profit-making activity or commercial gain

- You may freely distribute the URL identifying the publication in the public portal

If you believe that this document breaches copyright please contact us providing details, and we will remove access to the work immediately and investigate your claim 


\title{
Mirror Chern numbers in the hybrid Wannier representation
}

\author{
Tomáš Rauch, ${ }^{1}$ Thomas Olsen $\odot,{ }^{2}$ David Vanderbilt $\odot,{ }^{3}$ and Ivo Souza ${ }^{4,5}$ \\ ${ }^{1}$ Friedrich-Schiller-University Jena, 07743 Jena, Germany \\ ${ }^{2}$ Computational Atomic-Scale Materials Design, Department of Physics, Technical University of Denmark, 2800 Kgs. Lyngby, Denmark \\ ${ }^{3}$ Department of Physics and Astronomy, Rutgers University, Piscataway, New Jersey 08854-8019, USA \\ ${ }^{4}$ Centro de Física de Materiales, Universidad del País Vasco, 20018 San Sebastián, Spain \\ ${ }^{5}$ Ikerbasque Foundation, 48013 Bilbao, Spain
}

(Received 26 January 2021; accepted 16 April 2021; published 3 May 2021)

\begin{abstract}
The topology of electronic states in band insulators with mirror symmetry can be classified in two different ways. One is in terms of the mirror Chern number, an integer that counts the number of protected Dirac cones in the Brillouin zone of high-symmetry surfaces. The other is via a $\mathbb{Z}_{2}$ index that distinguishes between systems that have a nonzero quantized orbital magnetoelectric coupling ("axion-odd"), and those that do not ("axioneven"); this classification can also be induced by other symmetries in the magnetic point group, including time reversal and inversion. A systematic characterization of the axion $\mathbb{Z}_{2}$ topology has previously been obtained by representing the valence states in terms of hybrid Wannier functions localized along one chosen crystallographic direction, and inspecting the associated Wannier band structure. Here we focus on mirror symmetry, and extend that characterization to the mirror Chern number. We choose the direction orthogonal to the mirror plane as the Wannierization direction and show that the mirror Chern number can be determined from the winding numbers of the touching points between Wannier bands on mirror-invariant planes and from the Chern numbers of flat bands pinned to those planes. In this representation, the relation between the mirror Chern number and the axion $\mathbb{Z}_{2}$ index is readily established. The formalism is illustrated by means of $a b$ initio calculations for SnTe in the monolayer and bulk forms, complemented by tight-binding calculations for a toy model.
\end{abstract}

DOI: 10.1103/PhysRevB.103.195103

\section{INTRODUCTION}

The band theory of solids has been enriched in recent years by a vigorous study of its topological aspects. That effort resulted in a systematic topological classification of insulators on the basis of symmetry, and in the identification of a large number of topological materials. After an initial focus on the role of time-reversal symmetry, it was realized that crystallographic symmetries could also protect topological behaviors, leading to the notion of "topological crystalline insulators."

The assignment of an insulator to a particular topological class can be made by evaluating the corresponding topological invariant. Depending on the protecting symmetry, that invariant may assume one of two values $\left(\mathbb{Z}_{2}\right.$ classification), or it may assume any integer value ( $\mathbb{Z}$ classification). Other types of classifications such as $\mathbb{Z}_{4}$ also occur, but they do not concern us here. When the invariant vanishes the system is classified as trivial, and otherwise it is classified as nontrivial or topological. Topological insulators typically display robust gapless states at the boundary, which provide an experimental signature of topological behavior.

In some cases, the same symmetry may induce two different topological classifications. This happens, for example, with mirror symmetry, where a $\mathbb{Z}$ classification in terms of the mirror Chern number $(\mathrm{MCN})[1,2]$ coexists with a $\mathbb{Z}_{2}$ classification based on the quantized axion angle. The two classifications are not independent, and elucidating the relation between them is one goal of the present work.
The axion $\mathbb{Z}_{2}$ classification of three-dimensional (3D) insulators is based on the orbital magnetoelectric effect. In brief, the isotropic part of the linear orbital magnetoelectric tensor is conveniently expressed in terms of the axion angle $\theta$, which is defined only modulo $2 \pi$ as a bulk property. In the presence of "axion-odd" symmetries that flip its sign, the axion angle can assume only two values: $\theta=0$ (trivial), and $\theta=\pi$ (topological) [3-8].

The axion $\mathbb{Z}_{2}$ index was originally introduced for timereversal invariant insulators, where it was shown to be equivalent to the "strong" $\mathbb{Z}_{2}$ index $v_{0}=0$ or 1 , that is, $\theta=$ $\pi v_{0}$. More generally, axion-odd symmetries can be classified as proper rotations combined with time reversal (including time reversal itself), and improper rotations (including inversion and reflection) not combined with time reversal; in both cases, the associated symmetry operation in the magnetic space group may include a fractional translation. This results in a large number of magnetic space groups that can host axion-odd topological insulators. A recent realization is the $\mathrm{MnBi}_{2} \mathrm{Te}_{4}$ family of antiferromagnetic materials [7-9], whose axion topology is protected by the time-reversal operation combined with a half-lattice translation as envisioned in Ref. [10].

To aid the computational search for axionic topological insulators, it is useful to devise simple procedures for determining the (quantized) axion angle $\theta$. Unfortunately, subtle gauge issues make its direct evaluation from the valence Bloch states rather challenging in general [5]. Notable exceptions are 
centrosymmetric insulators, both nonmagnetic and magnetic. For such systems, the axion $\mathbb{Z}_{2}$ index can be obtained by counting the number of odd-parity states at high-symmetry points in the Brillouin zone (BZ) $[11,12]$.

Recently, an alternative procedure was introduced based on representing the valence states in terms of hybrid Wannier (HW) functions that are maximally localized along a chosen crystallographic direction $z$. The HW centers along $z$, also known as "Wilson-loop eigenvalues," form a band structure when plotted as a function of $k_{x}$ and $k_{y}$; in the presence of one or more axion-odd symmetries, the quantized $\theta$ value can be determined from this "Wannier band structure," often by mere visual inspection [13].

In the HW representation, axion-odd symmetries are naturally classified as " $z$-preserving" or " $z$-reversing," and the rules for deducing the axion $\mathbb{Z}_{2}$ index are different in each case (they also depend on whether or not the symmetry operation involves a fractional translation along $z$ ) [13]. Time reversal is an example of a $z$-preserving operation, while inversion is $z$ reversing. Mirror operations may be placed in one group or the other, depending on whether the Wannierization direction $z$ lies in the reflection plane (vertical mirror) or is orthogonal to it (horizontal mirror). In this work we make the latter choice, so that the mirror operation of interest becomes

$$
M_{z}: z \rightarrow-z
$$

which is manifestly $z$ reversing.

A simple mirror symmetry without a glide component protects not only the axion $\mathbb{Z}_{2}$ classification, but also a $\mathbb{Z}$ or $\mathbb{Z} \times \mathbb{Z}$ classification based on one or two MCNs, depending on the type of mirror. This raises the question of whether the HW representation might also be convenient for determining the MCNs, and for illuminating their relationship to the quantized axion angle.

In this work, we address the above questions by investigating in detail the Wannier bands in the presence of $M_{z}$ symmetry. We clarify the generic behaviors that are expected and discuss the rules for deducing the MCNs. By comparing those rules with the ones obtained in Ref. [13] for the axion $\mathbb{Z}_{2}$ index, we establish the relation between the two classifications.

The paper is organized as follows. In Sec. II we first distinguish between "type-1" and "type-2" crystallographic mirror operations; we then review the definitions of Chern invariants and MCNs in terms of the Bloch states in the filled bands; finally, we introduce maximally localized HW functions spanning the valence states, and assign Chern numbers to isolated groups of Wannier bands. This background material sets the stage for the developments in the remainder of the paper. In Sec. III we discuss the generic features of the Wannier band structure in the presence of $M_{z}$ symmetry, and obtain a relation between Chern numbers and winding numbers in groups of bands touching on a mirror plane. The rules for deducing the MCNs from the Chern numbers and winding numbers on the mirror planes are given in Sec. IV, where their relation to the quantized axion angle is also established. In Sec. V we describe the numerical methods that are used in Sec. VI to apply the formalism to several prototypical systems. We summarize and conclude in Sec. VII, and present in three
Appendixes some derivations that were left out of the main text.

\section{PRELIMINARIES}

\section{A. Two types of crystallographic mirrors}

We begin by observing that if a crystal is left invariant under an $M_{z}$ reflection operation, then its Bravais lattice must contain vectors pointing along $z$. To construct the shortest such vector $\mathbf{a}_{3}=c \hat{\mathbf{z}}$, we pick the shortest vector $\widetilde{\mathbf{a}}_{3}$ connecting lattice points on adjacent horizontal lattice planes. If $\widetilde{\mathbf{a}}_{3}$ points along $z$ then we take it as $\mathbf{a}_{3}$, and we say that the mirror is of type 1 . Otherwise we choose the vector $\mathbf{a}_{3}=\widetilde{\mathbf{a}}_{3}-M_{z} \widetilde{\mathbf{a}}_{3}$ connecting second-neighbor lattice planes, and the mirror is of type 2 .

The two types of crystallographic mirrors are exemplified in two dimensions in Fig. 1, where the mirror lines $z=0$ and $c / 2$ are labeled $\mathrm{A}$ and $\mathrm{B}$, and the reciprocal-space lines $k_{z}=0$ and $k_{z}=\pi / c$ are labeled $\mathrm{G}$ and $\mathrm{X}$. The same notation will be used in three dimensions, where $A$ and $B(G$ and $X)$ become planes in real (reciprocal) space.

The distinction between mirror operations that leave pointwise invariant two inequivalent planes in the BZ, and those that leave invariant only one BZ plane, was made in Refs. [14,15]. Since MCNs are defined on such planes [1,2], a $3 \mathrm{D}$ insulator with a type-1 mirror is characterized by two separate MCNs $\mu_{\mathrm{G}}$ and $\mu_{\mathrm{X}}$, while for a type- 2 mirror there is a single $\mathrm{MCN} \mu_{\mathrm{G}}$. If the crystallographic space group contains additional mirror operations, there will be additional MCNs associated with them.

\section{B. Chern invariants in band insulators}

\section{Generic insulators}

Before introducing MCNs for insulators with reflection symmetry, let us define Chern invariants for generic twodimensional (2D) and 3D band insulators in terms of the $\mathbf{k}$-space Berry curvature of the valence states [5].

In two dimensions the Berry curvature of a Bloch state $\left|\psi_{n \mathbf{k}}\right\rangle$ with cell-periodic part $\left|u_{n \mathbf{k}}\right\rangle$ is a scalar defined as

$$
\Omega_{n \mathbf{k}}=-2 \operatorname{Im}\left\langle\partial_{k_{x}} u_{n \mathbf{k}} \mid \partial_{k_{y}} u_{n \mathbf{k}}\right\rangle,
$$

where $\mathbf{k}=\left(k_{x}, k_{y}\right)$, and the Chern number is given by

$$
C=\frac{1}{2 \pi} \int_{2 \mathrm{DBZ}} \sum_{n=1}^{J} \Omega_{n \mathbf{k}} d^{2} k,
$$

where the summation is over the $J$ filled energy bands. Since the Berry curvature has units of length squared, $C$ is a dimensionless number, and for topological reasons it must be an integer. The Chern number is a global property of the manifold of occupied states, remaining invariant under multiband gauge transformations described by $J \times J$ unitary matrices at each $\mathbf{k}$, and it vanishes when the crystal has time-reversal symmetry. If a $2 \mathrm{D}$ magnetic crystal has a nonzero Chern number $C$, when that crystal is terminated at an edge there will be $|C|$ edge modes crossing the bulk gap, whose chirality will depend on the sign of $C$. 


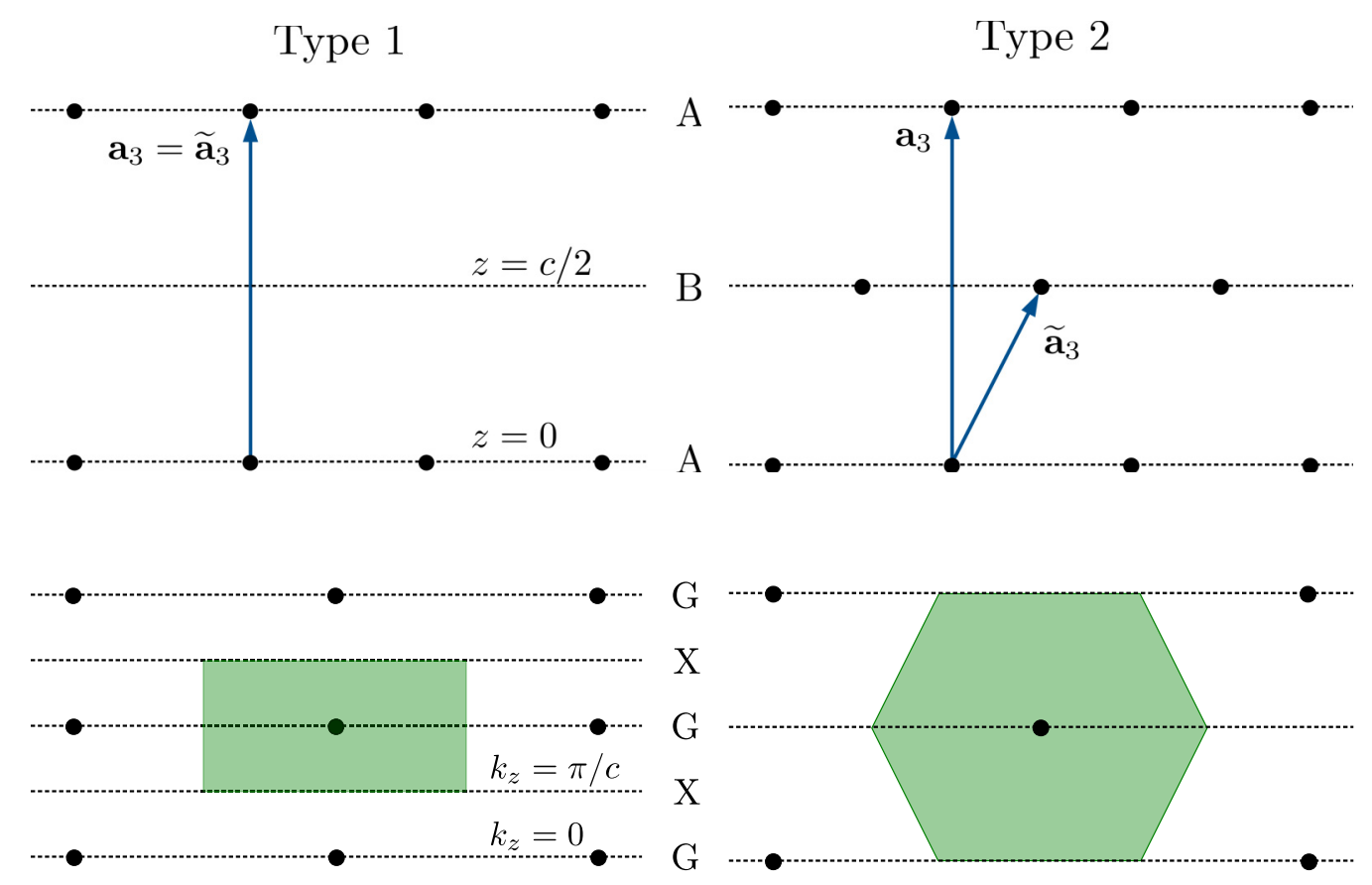

FIG. 1. The upper panel shows schematically a pair of 2D crystals lying on the $(x, z)$ plane; each has one atom per primitive cell (black dots), and lattice constant $c$ along $z$. The crystal on the left has a rectangular lattice and a type-1 horizontal mirror, with inequivalent mirror lines $z=0 \bmod c(\mathrm{~A})$ and $z=c / 2 \bmod c(\mathrm{~B})$, shown as dashed lines; the one on the right has a centered rectangular lattice and a type-2 mirror, with equivalent mirror lines $\mathrm{A}$ and $\mathrm{B}$. The lattice vectors $\mathbf{a}_{3}$ and $\widetilde{\mathbf{a}}_{3}$ are defined in the main text. The lower panel shows the reciprocal lattices, with a separation of $2 \pi / c$ between horizontal lattice lines G. On the left the periodicity along $k_{z}$ is $2 \pi / c$, and hence both $k_{z}=0$ mod $2 \pi / c$ (G) and $k_{z}=\pi / c \bmod 2 \pi / c(\mathrm{X})$ are pointwise-invariant mirror lines, as indicated by the dashed lines. On the right, where the periodicity along $k_{z}$ is $4 \pi / c, \mathrm{G}$ is a mirror-invariant line but $\mathrm{X}$ is not. The associated Brillouin zones are indicated by the shaded green areas.

Three-dimensional insulators are characterized by a Chern vector

$$
\mathbf{K}=\frac{1}{2 \pi} \int_{3 \mathrm{DBZ}} \sum_{n=1}^{J} \boldsymbol{\Omega}_{n \mathbf{k}} d^{3} k,
$$

where now $\mathbf{k}=\left(k_{x}, k_{y}, k_{z}\right)$ and the Berry curvature has become a vector field, $\boldsymbol{\Omega}_{n \mathbf{k}}=-\operatorname{Im}\left\langle\partial_{\mathbf{k}} u_{n \mathbf{k}}|\times| \partial_{\mathbf{k}} u_{n \mathbf{k}}\right\rangle$. The Chern vector has units of inverse length, and is quantized to be a reciprocal-lattice vector. Like the Chern number in two dimensions, the Chern vector always vanishes in nonmagnetic crystals.

Given a set of lattice vectors $\mathbf{a}_{j}$ and dual reciprocal-lattice vectors $\mathbf{b}_{j}$, the expansion $\mathbf{K}=\sum_{j} C_{j} \mathbf{b}_{j}$ defines a triad of integer Chern indices $C_{j}$. Let us orient the Cartesian axes such that $\mathbf{a}_{3}=c \hat{\mathbf{z}}$. The vectors $\mathbf{b}_{1}$ and $\mathbf{b}_{2}$ then lie on the $(x, y)$ plane, and the third Chern index can be expressed as

$$
C_{3}=\frac{c}{2 \pi} \int_{0}^{2 \pi / c} C\left(k_{z}\right) d k_{z}
$$

where

$$
C\left(k_{z}\right)=\frac{1}{2 \pi} \int_{2 \mathrm{DBZ}} \sum_{n=1}^{J} \Omega_{n}^{z}\left(k_{x}, k_{y}, k_{z}\right) d k_{x} d k_{y} .
$$

The integral in Eq. (6) is over a slice of the 3D BZ spanned by $\mathbf{b}_{1}$ and $\mathbf{b}_{2}$ at fixed $k_{z}$. By viewing it as an effective 2D $\mathrm{BZ}$ and comparing with Eq. (3), it becomes clear that $C\left(k_{z}\right)$ is a Chern number; and since in a gapped system its integer value cannot change with the continuous parameter $k_{z}$, Eq. (5) reduces to $C_{3}=C\left(k_{z}\right)$ evaluated at any $k_{z}$. The Chern indices of $3 \mathrm{D}$ insulators can therefore be evaluated as Chern numbers defined over individual BZ slices.

\section{Mirror-symmetric insulators}

We now consider a 3D crystalline insulator with mirror symmetry $M_{z}$, and assume that its Chern vector $\mathbf{K}$ vanishes. A new integer-valued topological index, the $\mathrm{MCN}$, can be defined for such a system as follows [1,2].

On the mirror-invariant BZ planes, $\mathrm{G}$ and possibly $\mathrm{X}$, the energy eigenstates are also eigenstates of $M_{z}$. The eigenvalues are $i^{F} p$, where $p= \pm 1$ is the "mirror parity" and $F=0$ or 1 when the electrons are treated as spinless or spinful particles, respectively. The occupied Bloch states on those planes can therefore be grouped into "even" $(p=+1)$ and "odd" $(p=$ $-1)$ sectors under reflection about the A plane $z=0$, each carrying its own Chern number. The Chern numbers of the two sectors on the $\mathrm{G}$ plane $k_{z}=0$ are given by

$$
C_{\mathrm{G}}^{ \pm}=\frac{1}{2 \pi} \int_{2 \mathrm{DBZ}} \sum_{n=1}^{J} f_{n \mathbf{k}}^{ \pm} \Omega_{n}^{z}\left(k_{x}, k_{y}, k_{z}=0\right) d k_{x} d k_{y},
$$

where $f_{n \mathbf{k}}^{+}=1-f_{n \mathbf{k}}^{-}$equals one or zero for a state with $p=$ \pm 1 , respectively. The MCN is defined as

$$
\mu_{\mathrm{G}}=\frac{1}{2}\left(C_{\mathrm{G}}^{+}-C_{\mathrm{G}}^{-}\right),
$$

and it is guaranteed to be an integer since $C_{\mathrm{G}}^{+}+C_{\mathrm{G}}^{-}=C_{3}$ vanishes by assumption. If the mirror is of type 1 , the plane $\mathrm{X}$ 
carries a second $\mathrm{MCN}$

$$
\mu_{\mathrm{X}}=\frac{1}{2}\left(C_{\mathrm{X}}^{+}-C_{\mathrm{X}}^{-}\right),
$$

where $C_{\mathrm{X}}^{ \pm}$is obtained by replacing $k_{z}=0$ with $k_{z}=\pi / c$ in Eq. (7). The MCNs remain invariant under multiband gauge transformations that do not mix the two mirror-parity sectors. When they are nonzero, protected gapless modes appear on surfaces that retain the mirror symmetry $M_{z}$, with $\left|\mu_{\mathrm{G}}\right|$ and $\left|\mu_{\mathrm{X}}\right|$ counting the number of Dirac cones on the two $M_{z^{-}}$ invariant lines in the surface BZ [16].

In the case of a $2 \mathrm{D}$ or quasi-2D insulator with reflection symmetry $M_{z}$ about its own plane, the entire $2 \mathrm{D} \mathrm{BZ}$ is left invariant under $M_{z}$. Such a system has a unique MCN

$$
\mu_{2 \mathrm{D}}=\frac{1}{2}\left(C_{+}-C_{-}\right) \text {, }
$$

where $C_{+}$and $C_{-}$are obtained by inserting the 2D Berry curvature of Eq. (2) in Eq. (7). When the net Chern number $C=C_{+}+C_{-}$vanishes, $\left|\mu_{2 \mathrm{D}}\right|$ becomes an integer that counts the number of pairs of counterpropagating chiral edge modes [17].

We note in passing that spin-orbit coupling is required to obtain non-vanishing MCNs in systems that are either nonmagnetic or whose magnetic order is collinear.

\section{The hybrid Wannier representation}

\section{Hybrid Wannier functions and Wannier bands}

HW functions are obtained from the valence Bloch states of a $2 \mathrm{D}$ or 3D crystalline insulator by carrying out the Wannier construction along a chosen reciprocal-lattice direction. They are therefore localized along one direction only, in contrast to ordinary Wannier functions which are localized in all spatial directions.

Let us momentarily return to a generic $3 \mathrm{D}$ insulating crystal, not necessarily mirror-symmetric. We denote by $z$ the chosen localization direction and let $\boldsymbol{\kappa}=\left(k_{x}, k_{y}\right)$, so that the wave vector in the $3 \mathrm{D} \mathrm{BZ}$ becomes $\mathbf{k}=\left(\boldsymbol{\kappa}, k_{z}\right)$. Given a gauge for the Bloch states that is periodic in $k_{z},\left|\psi_{n \kappa, k_{z}+2 \pi / c}\right\rangle=$ $\left|\psi_{n k k_{z}}\right\rangle$, the corresponding HW functions are defined as

$$
\left|h_{l n \kappa}\right\rangle=\frac{1}{2 \pi} \int_{-\pi / c}^{\pi / c} e^{-i k_{z} l c} e^{-i \kappa \cdot \mathbf{r}}\left|\psi_{n \kappa k_{z}}\right\rangle d k_{z},
$$

where the index $l$ runs over unit cells along $z$, and $n$ runs over the $J$ HW functions in one unit cell. By factoring out $e^{-i \kappa \cdot \mathbf{r}}$, we have made the HW functions cell periodic in the in-plane directions, $h_{l n \kappa}(\mathbf{r}+\mathbf{R})=h_{l n \kappa}(\mathbf{r})$ for any in-plane lattice vector $\mathbf{R}$. This will be convenient later on when we define Berry curvatures and Chern numbers in the HW representation.

For each $\boldsymbol{\kappa}$ in the projected 2D BZ, we choose the multiband gauge for the Bloch states in such a way that the HW functions have the smallest possible quadratic spread along $z$. Such maximally localized HW functions satisfy the eigenvalue equation [18]

$$
P_{\kappa} z P_{\kappa}\left|h_{l n \kappa}\right\rangle=z_{l n \kappa}\left|h_{l n \kappa}\right\rangle,
$$

where $P_{\kappa}$ is the projection operator onto the space of valence states with in-plane wave vector $\boldsymbol{\kappa}$. The eigenvalues in Eq. (12) are the HW centers

$$
z_{\text {ln }}=\left\langle h_{l n \kappa}|z| h_{l n \kappa}\right\rangle,
$$

which form Wannier bands. These are periodic in real space along $z$, as well as in the in-plane reciprocal space,

$$
z_{l n \kappa}=z_{0 n \kappa}+l c, \quad z_{l n, \kappa+\mathbf{G}}=z_{l n \kappa},
$$

where $\mathbf{G}$ is an in-plane reciprocal lattice vector.

A Wannier band structure is said to be gapped if it contains at least one Wannier band per vertical cell that is separated from the band below by a finite gap at all $\boldsymbol{\kappa}$. When that is the case, we choose the cell contents in such a way that the first band, $n=1$, has a gap below it.

\section{Chern numbers of Wannier bands}

The Berry curvature of a HW state is defined as

$$
\Omega_{l n}=-2 \operatorname{Im}\left\langle\partial_{k_{x}} h_{l n} \mid \partial_{k_{y}} h_{l n}\right\rangle,
$$

and periodicity along $z$ implies that $\Omega_{l n}=\Omega_{0 n}$. (Here and in the following, we will frequently drop the index $\boldsymbol{\kappa}$.) When the Wannier spectrum is gapped, it becomes possible to associate a Chern number with each isolated group $a$ of bands within a vertical cell,

$$
C_{l a}=\frac{1}{2 \pi} \int_{2 \mathrm{DBZ}} \sum_{n \in a} \Omega_{l n} d^{2} k=C_{0 a} .
$$

From the HW states in a given group, one can construct Bloch-like states at any $\mathbf{k}=\left(k_{x}, k_{y}, k_{z}\right)$ by inverting Eq. (11). In general these are not energy eigenstates, and their band indices label Wannier bands rather than energy bands. Their Berry curvatures along $z$ are given by

$$
\Omega_{n}^{z}\left(k_{x}, k_{y}, k_{z}\right)=\sum_{l} e^{i k_{z} l c} \Omega_{0 n, l n}\left(k_{x}, k_{y}\right),
$$

where

$$
\Omega_{0 n, l n}=i\left\langle\partial_{k_{x}} h_{0 n} \mid \partial_{k_{y}} h_{l n}\right\rangle-i\left\langle\partial_{k_{y}} h_{0 n} \mid \partial_{k_{x}} h_{l n}\right\rangle
$$

is a matrix generalization of Eq. (15) [19]. To evaluate the net Chern number $C_{a}\left(k_{z}\right)$ of that group of Bloch-like states on a slice of the 3D BZ, we insert Eq. (17) in Eq. (6) and restrict the summation over $n$ to $n \in a$. The contributions from the $l \neq 0$ terms drop out, ${ }^{1}$ yielding

$$
C_{a}\left(k_{z}\right)=C_{0 a} .
$$

Hence the Chern numbers are the same in the Bloch-like and HW representations, as expected since the two representations are related by a unitary transformation. When the group $a$ comprises all $J$ Wannier bands in one vertical cell, its Chern number becomes equal to the Chern index $C_{3}$ of Eq. (5), which vanishes by assumption.

\footnotetext{
${ }^{1}$ The expression for $C_{a}\left(k_{z}\right)$ involves $\int_{0}^{2 \pi / a} \partial_{k_{x}} Y_{0 n, l n}\left(k_{x}\right) d k_{x}$ where $Y_{0 n, l n}\left(k_{x}\right)=\int_{0}^{2 \pi / b} A_{0 n, l n}^{y}\left(k_{x}, k_{y}\right) d k_{y}$, and another similar integral $\int_{0}^{2 \pi / b} \partial_{k_{y}} X_{0 n, l n}\left(k_{y}\right) d k_{y}$. When $l \neq 0$ the quantity $Y_{0 n, l n}\left(k_{x}\right)$ becomes fully invariant under band-diagonal gauge transformations of the $\mathrm{HW}$ states. Hence its value at $k_{x}=2 \pi / a$ must be the same as at $k_{x}=0$, and the integral vanishes.
} 


\section{MIRROR-SYMMETRIC WANNIER BANDS}

With the above background material in hand, we now return to our system of interest-a 3D insulator with $M_{z}$ symmetry-and construct HW functions localized along the direction $z$ orthogonal to the mirror plane. We begin this section by discussing the generic features of Wannier band structures with $M_{z}$ symmetry.

\section{A. Flat versus dispersive bands and the uniform parity assumption}

If $M_{z}$ is a symmetry of the system, the operator $P z P$ anticommutes with $M_{z}$. It follows that if a HW function $\left|h_{l n}\right\rangle$ satisfies Eq. (12) with eigenvalue $z_{l n}, M_{z}\left|h_{l n}\right\rangle$ satisfies it with eigenvalue $-z_{l n}$. Since $z_{l n}$ is defined only modulo $c$, two situations may occur. (i) $\left|h_{l n}\right\rangle$ and $M_{z}\left|h_{l n}\right\rangle$ are orthogonal, in which case a pair of dispersive bands appear at $\pm z_{l n}$. (ii) $\left|h_{l n}\right\rangle$ and $M_{z}\left|h_{l n}\right\rangle$ are the same up to a phase, in which case $\left|h_{l n}\right\rangle$ is an eigenstate of $M_{z}$, and a single flat band appears at either $z=0$ (A plane) or $z=c / 2$ (B plane). The Wannier bands of the system can therefore be classified into flat bands of even or odd mirror parity at A; flat bands of even or odd mirror parity at $\mathrm{B}$; and dispersive pairs appearing at $\pm z$.

If there are several flat bands on a given mirror plane and not all of them have the same parity, those of opposite parity will generally have a nonzero $P z P$ matrix element between them, and will tend to hybridize and split to form dispersive pairs. Thus, all flat bands pinned at $\mathrm{A}$ are expected to have the same parity $p_{\mathrm{A}}$, and all flat bands pinned at $\mathrm{B}$ are expected to have the same parity $p_{\mathrm{B}}$. Following Ref. [13], we call this the "uniform parity" assumption. As discussed in Ref. [13], this assumption is closely related to a well-known theorem on the minimum number of zero-energy modes in bipartite lattices [20-22].

Under the uniform parity assumption, the numbers $\bar{N}_{\mathrm{A}}$ and $\bar{N}_{\text {B }}$ of flat bands at A and B can be expressed in terms of the imbalance between even- and odd-parity valence Bloch states at the mirror-invariant plane(s) in the BZ. For a type-1 mirror we have

$$
\bar{N}_{\mathrm{A}}=\frac{1}{2}\left|\Delta N_{\mathrm{G}}+\Delta N_{\mathrm{X}}\right|
$$

and

$$
\bar{N}_{\mathrm{B}}=\frac{1}{2}\left|\Delta N_{\mathrm{G}}-\Delta N_{\mathrm{X}}\right|,
$$

where $\Delta N_{\mathrm{G}}$ and $\Delta N_{\mathrm{X}}$ denote the excess of even over odd valence states at $G$ and $X$, respectively. Hence if the mirrorparity content is balanced at both $\mathrm{G}$ and $\mathrm{X}$, flat Wannier bands are absent from both A and B; if it is balanced only at $\mathrm{G}$ but not at $X$ or vice versa, the same number of flat bands is present at $\mathrm{A}$ and at $\mathrm{B}$; and if it is unbalanced at both $\mathrm{G}$ and $\mathrm{X}$, the number of flat bands at $\mathrm{B}$ can differ from the number at $\mathrm{A}$. The corresponding relation for a type- 2 mirror is

$$
\bar{N}_{\mathrm{A}}=\bar{N}_{\mathrm{B}}=\frac{1}{2}\left|\Delta N_{\mathrm{G}}\right| .
$$

Equations (20)-(22) are derived in Appendix A.

\section{B. Types of generic degeneracies}

In this section, we consider the types of degeneracies that are typical of the Wannier spectra of insulators with $M_{z}$ symmetry. We call a degeneracy generic when it occurs without the assistance of any symmetries other than $M_{z}$. If in addition the degeneracy is codimension protected, we call it accidental.

Accidental degeneracies away from the A and B planes have codimension three, and hence they require fine tuning. On the mirror planes, there are two types of generic degeneracies: multiple flat bands pinned to the same plane, and accidental touchings, at isolated points in the 2D BZ, between one or more pairs of dispersive bands. Other possibilities such as nodal lines are nongeneric and will not be considered further. In the following we focus on the A plane $z=0$, but the discussion would be identical for the $\mathrm{B}$ plane $z=c / 2$.

\section{Point nodes between pairs of dispersive bands}

If there are no flat bands pinned at $z=0$, any bands near $z=0$ must come in dispersive pairs at $\pm z$. If there is a single pair, we construct from the two HW functions at each $\kappa$ a pair of orthogonal states with opposite parities about $z=0$. In this basis, the $z$ operator is represented by a matrix of the form

$$
\left(\begin{array}{cc}
0 & f_{\kappa} \\
f_{\kappa}^{*} & 0
\end{array}\right)
$$

with eigenvalues $z_{\kappa}= \pm\left|f_{\kappa}\right|$. The two bands touch at $z=0$ when $\left|f_{\kappa}\right|=0$, and for that to happen both the real and imaginary parts of $f_{\kappa}$ must vanish; this means that such degeneracies have codimension two, and hence they occur at isolated points in the 2D BZ. (When the bands disperse linearly close to the nodal point, the degeneracy is called a "Dirac node.") If more than one dispersive band pair is involved, $f_{\kappa}$ becomes a matrix. The degeneracy condition $\operatorname{det}\left(f_{\kappa}\right)=0$ again leads to point nodes on the $z=0$ plane. Generically, these are simple nodes where only two bands meet. However, with additional symmetries or fine tuning, more than one pair of bands may become degenerate at a given node.

In summary, pairs of dispersive Wannier bands can touch accidentally at isolated points on a mirror plane free of flat bands. We note that the same happens, and for the same mathematical reasons, with the energy bands of models with sublattice symmetry [22].

\section{Flat bands repel point nodes}

When one or more flat bands are present at $z=0$, they gap out the point nodes. Let us show this for the simplest case of one flat band surrounded by a dispersive pair. Choosing a basis of $M_{z}$ eigenstates within this three-band space, the matrix representation of the $z$ operator takes the form

$$
\left(\begin{array}{ccc}
0 & f_{\kappa} & g_{\kappa} \\
f_{\kappa}^{*} & 0 & 0 \\
g_{\kappa}^{*} & 0 & 0
\end{array}\right),
$$

where we have chosen the first basis state to have the opposite mirror parity from the other two. The eigenvalues are $z_{\kappa}=0$ (flat band) and $z_{\kappa}= \pm \sqrt{\left|f_{\kappa}\right|^{2}+\left|g_{\kappa}\right|^{2}}$ (dispersive pair). An accidental degeneracy between the pair requires the real and imaginary parts of both $f_{\kappa}$ and $g_{\kappa}$ to vanish (codimension four). In general this cannot be achieved by adjusting $\kappa$ alone; it also requires fine tuning the parameters $f_{\kappa}$ and $g_{\kappa}$. 
In conclusion, flat bands and point nodes do not generally coexist on a mirror plane. Although we have shown this only for the case of one flat band plus one dispersive pair, the same result is expected to hold when several flat bands and/or dispersive pairs are present. That scenario has in fact been considered for the analogous problem of energy bands in models with sublattice symmetry [22].

\section{Spinful time-reversal symmetry excludes flat bands}

The presence of flat bands on the mirror planes can sometimes be ruled out on the basis of symmetry. This is the case for a crystal that has both $M_{z}$ symmetry and spinful time-reversal symmetry $\mathcal{T}$. Since $\left[P_{\kappa} z P_{\kappa}, \mathcal{T}\right]=0$, the standard Kramers-degeneracy argument applies to the Wannier bands: if $\left|h_{\kappa}\right\rangle$ is an eigenstate of $P_{\kappa} z P_{\kappa}$ with eigenvalue $z_{\kappa}$, then $\left|h_{-\kappa}^{\prime}\right\rangle=\mathcal{T}\left|h_{\kappa}\right\rangle$ is an orthogonal eigenstate with the same eigenvalue. Now suppose that $\left|h_{\kappa}\right\rangle$ is a flat-band state at A, with $M_{z}$ eigenvalue $\lambda= \pm i$. Then $\left|h_{-\kappa}^{\prime}\right\rangle$ is also a flat-band state, and using $\left[M_{z}, \mathcal{T}\right]=0$ we find that its mirror eigenvalue is $\lambda^{*}=-\lambda$. Since the two flat bands have opposite mirror eigenvalues, they will generally hybridize to form a dispersive pair.

Another example is a crystal that has both $M_{z}$ symmetry, and spinful $\mathcal{T}$ combined with inversion $\mathcal{I}$. The combined symmetry $\mathcal{I} * \mathcal{T}$ renders the energy bands Kramers-degenerate at every $\mathbf{k}$, and since $\left[M_{z}, \mathcal{I} * \mathcal{T}\right]=0$ and $M_{z}$ has purely imaginary eigenvalues, Kramers pairs of Hamiltonian eigenstates on the invariant BZ planes have opposite $M_{z}$ eigenvalues. The mirror-parity content is therefore balanced on those planes, and from Eqs. (20)-(22) we conclude that both $\bar{N}_{\mathrm{A}}$ and $\bar{N}_{\mathrm{B}}$ vanish. (Note that while the energy bands are Kramers degenerate in the presence of $\mathcal{I} * \mathcal{T}$ symmetry, the Wannier bands are not. The difference is that $\mathcal{I} * \mathcal{T}$ commutes with the Hamiltonian, but it anticommutes with $P z P$.)

In summary, spinful time-reversal symmetry, either by itself or in combination with inversion, rules out the presence of flat Wannier bands on the mirror planes (under the uniform parity assumption).

\section{Chern numbers in gapped band structures}

When an $M_{z}$-symmetric Wannier band structure is gapped, the $J$ bands per cell can be grouped into three internally connected collections [13]: one containing bands that are pinned at $\mathrm{A}$ (over the entire 2D BZ or at isolated $\boldsymbol{\kappa}$ points), another containing bands that are pinned at $\mathrm{B}$, and a third containing "unpinned" bands, in the sense that they do not touch the mirror planes anywhere in the 2D BZ. In Ref. [13] these three collections were called origin-centered, boundary-centered, and uncentered, respectively.

Letting $\alpha=\mathrm{A}$ or $\mathrm{B}$, in each vertical cell $l$ there are in general

(1) $\bar{N}_{\alpha^{+}}$flat bands at $\alpha$ of even parity

(2) $\bar{N}_{\alpha^{-}}$flat bands at $\alpha$ of odd parity

(3) $\widetilde{N}_{\alpha}$ dispersive bands touching at $\alpha$.

In the $\alpha$-pinned collection, and $\widetilde{N}_{\mathrm{UC}}$ dispersive bands in the unpinned collection. (At this stage we do not yet assume uniform parity for the flat bands, nor do we invoke the fact that flat bands repel point nodes.) In the home cell $l=0$, the dispersive bands in the A-pinned collection come in pairs at $\pm z$, and those in the B-pinned collection come in pairs at $z$ and $c-z$. In the case of the unpinned collection we have a choice, since the mirror-symmetric partners never become degenerate; for definiteness, we choose the contents of the home cell so that the bands in the unpinned collection come in pairs at $\pm z$.

For each of the seven groups listed above, we can add up the Chern numbers in that group to get $\bar{C}_{\alpha^{ \pm}}, \widetilde{C}_{\alpha}$, and $\widetilde{C}_{\mathrm{UC}}$, keeping in mind that their sum $C_{3}$ vanishes by assumption,

$$
C_{\mathrm{A}}+C_{\mathrm{B}}+\widetilde{C}_{\mathrm{UC}}=0,
$$

where $C_{\alpha}=\bar{C}_{\alpha^{+}}+\bar{C}_{\alpha^{-}}+\widetilde{C}_{\alpha}$ is the net Chern number of the $\alpha$-pinned collection. We further decompose each of the three dispersive band subspaces into even and odd sectors under reflection about their centers, and assign separate Chern numbers to them,

$$
\begin{aligned}
\widetilde{C}_{\alpha} & =\widetilde{C}_{\alpha^{+}}+\widetilde{C}_{\alpha^{-}}, \\
\widetilde{C}_{\mathrm{UC}} & =\widetilde{C}_{\mathrm{UC}^{+}}+\widetilde{C}_{\mathrm{UC}^{-}} .
\end{aligned}
$$

In Appendix B we show that

$$
\widetilde{C}_{\alpha^{+}}-\widetilde{C}_{\alpha^{-}}=W_{\alpha},
$$

where $W_{\alpha}$ is the sum of the winding numbers of all the nodal points in the projected 2D BZ on the $\alpha$ mirror plane.

The winding number of a nodal point $\boldsymbol{\kappa}_{j}$ is defined as [23]

$$
W_{j}=\frac{1}{2 \pi} \oint_{c_{j}} \partial_{\kappa} \gamma_{\kappa} \cdot d \kappa,
$$

where the integral is over a small circle around the node. $W_{j}$ is an integer, typically taking values \pm 1 according to how the phase $\gamma_{\kappa}$ changes going around the node. In the simplest case where a single pair of bands meet at the node, $\gamma_{\kappa}$ is the phase angle of the complex matrix element $f_{\mathbf{k}}$ appearing in Eq. (23). If two or more pairs of bands meet at a node, $f_{\mathbf{k}}$ becomes a matrix and $\gamma_{\kappa}$ becomes the phase angle of its determinant (see Sec. V C).

Combining Eqs. (26a) and (27) we obtain

$$
W_{\alpha}=\widetilde{C}_{\alpha}-2 \widetilde{C}_{\alpha^{-}},
$$

which shows that $\widetilde{C}_{\alpha}$ has the same even or odd parity as $W_{\alpha}$. Since band pairs in the unpinned collection do not touch on the special planes, by applying the same argument in Appendix B that leads to Eq. (27) we obtain

$$
\widetilde{C}_{\mathrm{UC}^{+}}=\widetilde{C}_{\mathrm{UC}^{-}},
$$

which implies that their sum $\widetilde{C}_{\mathrm{UC}}$ is always an even number. ${ }^{2}$

\section{MIRROR CHERN NUMBERS IN THE HYBRID WANNIER REPRESENTATION}

We are finally ready to evaluate the MCNs in the HW representation, and then relate them to the axion $\mathbb{Z}_{2}$ index. In Sec. IV A we consider the case of a gapped Wannier spectrum, and in Sec. IV B we treat the gapless case.

\footnotetext{
${ }^{2}$ The fact that $\widetilde{C}_{\mathrm{UC}}$ is even can also be seen as follows [13]. The unpinned collection is formed by two disconnected groups of bands related by $M_{z}$ symmetry, which imposes the same Berry curvature at every $\kappa$ in the two groups, and hence the same Chern number.
} 
TABLE I. Parities under a type-1 mirror $M_{z}$ of Bloch-like states constructed from HW functions that are maximally localized along $z$. For spinful electrons, the parity is said to be "even" or "odd" when the $M_{z}$ eigenvalue is $+i$ or $-i$.

$$
\begin{aligned}
& \text { Bloch representation } \\
& \left.\mathrm{G}^{+}=\text {even about } \mathrm{A} \text { (and even about } \mathrm{B}\right) \\
& \mathrm{G}^{-}=\text {odd about } \mathrm{A}(\text { and odd about } \mathrm{B}) \\
& \mathrm{X}^{+}=\text {even about } \mathrm{A}(\text { and odd about } \mathrm{B}) \\
& \mathrm{X}^{-}=\text {odd about } \mathrm{A}(\text { and even about } \mathrm{B})
\end{aligned}
$$

Hybrid Wannier representation

$$
\begin{aligned}
& \mathrm{A}^{+}=\text {even about } \mathrm{A} \text {, generates } \mathrm{G}^{+} \text {and } \mathrm{X}^{+} \\
& \mathrm{A}^{-}=\text {odd about } \mathrm{A} \text {, generates } \mathrm{G}^{-} \text {and } \mathrm{X}^{-} \\
& \mathrm{B}^{+}=\text {even about } \mathrm{B} \text {, generates } \mathrm{G}^{+} \text {and } \mathrm{X}^{-} \\
& \mathrm{B}^{-}=\text {odd about } \mathrm{B}, \text { generates } \mathrm{G}^{-} \text {and } \mathrm{X}^{+} \\
& \text {Pairs } \mathrm{C} \text { and } \mathrm{C}^{\prime}, \quad \text { generates } \mathrm{G}^{+} \mathrm{G}^{-} \text {and } \mathrm{X}^{+} \mathrm{X}^{-}
\end{aligned}
$$

\section{A. Gapped Wannier band structure}

To recap, a generic gapped Wannier band structure with $M_{z}$ symmetry consists of seven band collections per cell. The four that are flat have well-defined mirror parities, and the three that are dispersive can be decomposed into even and odd sectors. This yields a total of $10 \mathrm{HW}$ groups with well-defined parities, each carrying its own Chern number.

\section{Type-1 mirrors}

To evaluate the MCNs $\mu_{\mathrm{G}}$ and $\mu_{\mathrm{X}}$, we construct from each of the $10 \mathrm{HW}$ groups a group of Bloch-like states by performing Bloch sums along $z$, and recall from Eq. (19) that their Chern numbers on any constant $k_{z}$ BZ slice (and, in particular, at $\mathrm{G}$ and $\mathrm{X}$ ) are the same as the Chern numbers of the parent HW groups. The final needed ingredient is Table I, which gives the mirror parities at $\mathrm{G}$ and $\mathrm{X}$ of the Bloch groups coming from each of the HW groups. This table is valid for both spinless and spinful mirror symmetry $M_{z}$, and it agrees with the parity rules for inversion symmetry $\mathcal{I}$ in one dimension [13]; this is consistent with the fact that $M_{z}=\mathcal{I} * C_{2}^{z}$ acts along $z$ in the same way as $\mathcal{I}$.

To evaluate $\mu_{\mathrm{G}}$, we need to split the occupied Bloch space at $\mathrm{G}$ into even- and odd-parity sectors about A. According to Table I, their Chern numbers are

$$
C_{\mathrm{G}}^{ \pm}=\left(\bar{C}_{\mathrm{A}^{ \pm}}+\widetilde{C}_{\mathrm{A}^{ \pm}}+\widetilde{C}_{\mathrm{UC}}^{ \pm}\right)+\left(\bar{C}_{\mathrm{B}^{ \pm}}+\widetilde{C}_{\mathrm{B}^{ \pm}}\right),
$$

where the first and second groups of terms correspond to Wannier groups that are even or odd about $\mathrm{A}$ and $\mathrm{B}$, respectively. Inserting this expression into Eq. (8) for $\mu_{\mathrm{G}}$ and then using Eqs. (27) and (30), we find

$$
2 \mu_{\mathrm{G}}=\left(\bar{C}_{\mathrm{A}^{+}}-\bar{C}_{\mathrm{A}^{-}}\right)+\left(\bar{C}_{\mathrm{B}^{+}}-\bar{C}_{\mathrm{B}^{-}}\right)+W_{\mathrm{A}}+W_{\mathrm{B}} .
$$

Under the uniform parity assumption the first group of terms becomes $p_{\mathrm{A}} \bar{C}_{\mathrm{A}}$, where $\bar{C}_{\mathrm{A}}$ is the total Chern number of the flat bands at $\mathrm{A}$, all of the same parity $p_{\mathrm{A}}= \pm 1$; similarly, the second group becomes $p_{\mathrm{B}} \bar{C}_{\mathrm{B}}$. Thus we arrive at

$$
\mu_{\mathrm{G}}=\frac{1}{2}\left(p_{\mathrm{A}} \bar{C}_{\mathrm{A}}+W_{\mathrm{A}}\right)+\frac{1}{2}\left(p_{\mathrm{B}} \bar{C}_{\mathrm{B}}+W_{\mathrm{B}}\right),
$$

and via similar steps Eq. (9) for $\mu_{X}$ turns into

$$
\mu_{\mathrm{X}}=\frac{1}{2}\left(p_{\mathrm{A}} \bar{C}_{\mathrm{A}}+W_{\mathrm{A}}\right)-\frac{1}{2}\left(p_{\mathrm{B}} \bar{C}_{\mathrm{B}}+W_{\mathrm{B}}\right) .
$$

Out of the three collections in a type-1 disconnected band structure, the uncentered collection does not contribute to the MCNs; and the A-centered and B-centered ones contribute as in Eqs. (33) and (34).

Equations (33) and (34) are a central result of this work, and in the following sections we will extract several conclusions from them. In practical applications, those equations can often be simplified: since flat bands and point nodes do not generically coexist on the mirror planes, at least one of the two terms inside each pair of parentheses will typically vanish.

Before proceeding, let us verify that Eq. (33) correctly yields an integer value for $\mu_{\mathrm{G}}$ when $C_{3}=0$. First we eliminate the winding numbers from Eq. (33) with the help of Eq. (29), and then we take mod 2 on both sides of the resulting equation to find

$$
\begin{aligned}
2 \mu_{\mathrm{G}} \bmod 2 & =\left(\bar{C}_{\mathrm{A}}+\widetilde{C}_{\mathrm{A}}+\bar{C}_{\mathrm{B}}+\widetilde{C}_{\mathrm{B}}\right) \bmod 2 \\
& =-\widetilde{C}_{\mathrm{UC}} \bmod 2,
\end{aligned}
$$

where Eq. (25) was used to go from the first to the second line. Given that $\widetilde{C}_{\mathrm{UC}}$ is an even number, we conclude that $\mu_{\mathrm{G}}$ is an integer. The proof is identical for Eq. (34).

We emphasize that the separate contributions from the Aand B-centered collection to Eqs. (33) and (34) are not always integer-valued. As can be seen from Eq. (37) below, those contributions assume half-integer values when the axion angle is quantized to $\theta=\pi$ by mirror symmetry; a concrete example where this happens will be given in Sec. VIC.

\section{Relation to the quantized axion coupling}

As mentioned in the Introduction, mirror symmetry belongs to the group of "axion-odd" symmetries that reverse the sign of the axion angle $\theta$. When one or more such symmetries are present in a $3 \mathrm{D}$ insulator with a vanishing Chern vector, $\theta$ is restricted to be zero or $\pi \bmod 2 \pi$, becoming a $\mathbb{Z}_{2}$ topological index.

In the case of mirror symmetry, where the band topology is already characterized by the MCNs, there should be a relation between them and the quantized $\theta$ value. Below we derive that relation for an insulator with a type-1 mirror and a gapped Wannier spectrum. To that end, we make use of the formalism of Ref. [13] for expressing $\theta$ in the HW representation.

First we write $\mu_{\mathrm{G}}+\mu_{\mathrm{X}}$ by combining Eqs. (33) and (34), and eliminate the winding numbers using Eq. (29). Then we take $\bmod 2$ on both sides to find

$$
\left(\mu_{\mathrm{G}}+\mu_{\mathrm{X}}\right) \bmod 2=C_{\mathrm{A}} \bmod 2 .
$$

Comparing with the relation $\theta / \pi=C_{\mathrm{A}} \bmod 2$ [13], valid for a gapped spectrum in the presence of a $z$-reversing axion-odd symmetry such as $M_{z}$, we conclude that

$$
\frac{\theta}{\pi}=\left(\mu_{\mathrm{G}}+\mu_{\mathrm{X}}\right) \bmod 2 .
$$

Thus, the system is axion-even $(\theta=0)$ or axion-odd $(\theta=\pi)$ depending on whether the sum of the two MCNs associated with $M_{z}$ is even or odd. Previously, this result had been inferred from an argument based on counting Dirac cones in the surface BZ [14,15]. Here, we have obtained it directly as a formal relation between bulk quantities expressed in the HW 
representation. As we will see shortly, the same relation holds when the Wannier spectrum is gapless.

\section{Type-2 mirrors}

In a crystal with a type- 2 mirror, where the planes $A$ and $B$ are equivalent and $G$ is the only mirror-invariant plane in reciprocal space, the unique $\mathrm{MCN} \mu_{\mathrm{G}}$ is obtained by setting $p_{\mathrm{B}}=p_{\mathrm{A}}, \bar{C}_{\mathrm{B}}=\bar{C}_{\mathrm{A}}$, and $W_{\mathrm{B}}=W_{\mathrm{A}}$ in Eq. (33),

$$
\mu_{\mathrm{G}}=p_{\mathrm{A}} \bar{C}_{\mathrm{A}}+W_{\mathrm{A}} \text {. }
$$

If flat bands are present at $\mathrm{A}$, they repel the point nodes. Hence $W_{\mathrm{A}}=0$, and therefore $\left|\mu_{\mathrm{G}}\right|=\left|\bar{C}_{\mathrm{A}}\right|$. Interestingly, in this case the magnitude of the MCN does not depend on the parity of the flat-band states; this simplifies considerably its numerical evaluation, since one does not need to know how the basis orbitals transform under $M_{z}$. Given that only the magnitude (not the sign) of the MCN is needed to establish the bulk-boundary correspondence, this is a potentially useful result.

Inserting Eq. (29) for $W_{\mathrm{A}}$ in Eq. (38), taking mod 2 on both sides, and again comparing with $\theta / \pi=C_{\mathrm{A}} \bmod 2$, we conclude that in this case the relation between the axion $\mathbb{Z}_{2}$ index and the MCN reads

$$
\frac{\theta}{\pi}=\mu_{\mathrm{G}} \bmod 2
$$

as stated in Ref. [15].

\section{Weakly coupled layered crystals}

Consider a crystal composed of weakly coupled identical layers that remain invariant under reflection about their own planes. Following Ref. [24], we assume that the layers are stacked exactly vertically. In this case the reflection symmetry about the individual layers becomes a type-1 mirror of the 3D structure, with two separate MCNs $\mu_{\mathrm{G}}$ and $\mu_{\mathrm{X}}$. In the fully decoupled limit where there is no $k_{z}$ dependence the $\mathrm{G}$ and $\mathrm{X}$ reciprocal planes become equivalent, so that $\mu_{\mathrm{X}}=\mu_{\mathrm{G}} \equiv \mu_{2 \mathrm{D}}$ where $\mu_{2 \mathrm{D}}$ is the MCN of an isolated layer [Eq. (10)]. But since the MCNs are integers, they cannot change if a weak interlayer coupling is introduced, and from Eqs. (33) and (34) we obtain

$$
\mu_{2 \mathrm{D}}=\frac{1}{2}\left(p_{\mathrm{A}} \bar{C}_{\mathrm{A}}+W_{\mathrm{A}}\right)
$$

for the unique MCN of a weakly coupled layered crystal.

If flat bands are present at A (the plane of a layer), then $W_{\mathrm{A}}=0$ and the net Chern number of the valence bands becomes $\bar{C}_{\mathrm{A}}+\widetilde{C}_{\mathrm{UC}} ;$ since the net Chern number vanishes by assumption and $\widetilde{C}_{\mathrm{UC}}$ is even, $\mu_{2 \mathrm{D}}=p_{\mathrm{A}} \bar{C}_{\mathrm{A}} / 2$ is clearly an integer. In this case $\left|\mu_{2 \mathrm{D}}\right|$ can be determined without knowing the parity of the flat-band states, as in the case of a type- 2 mirror with flat bands.

Let us now evaluate the axion $\mathbb{Z}_{2}$ index. Since $\mu_{\mathrm{G}}+\mu_{\mathrm{X}}=$ $2 \mu_{2 \mathrm{D}}$ is an even number, Eq. (37) yields

$$
\theta=0 \bmod 2 \pi \text {. }
$$

This is consistent with the assertion made in Ref. [24] that weakly coupled layered topological crystalline insulators are analogous to "weak topological insulators" with a vanishing strong $\mathbb{Z}_{2}$ invariant $v_{0}$.

\section{B. Gapless Wannier band structure}

Let us now apply our formalism to a $M_{z}$-symmetric system with a gapless Wannier spectrum. We start out by noting that such a spectrum must have degeneracies at both A and B. On those special planes the codimension is two, so point nodes are allowed. Flat bands can be ruled out since they would repel any nodes and generate a gap, and we assume that nodal lines are absent as well.

We are left with a scenario where there are point nodes at both $\mathrm{A}$ and $\mathrm{B}$, and these are connected by Wannier bands. The only way this can happen without the assistance of other symmetries is if there are only two Wannier bands, one in each half unit cell, since otherwise there is generically a gap somewhere in each half cell (accidental degeneracies away from $\mathrm{A}$ and $\mathrm{B}$ are not protected, since the codimension is three). With the assistance of other symmetries, the gapless spectrum may contain more than two bands per cell.

To treat the above scenario, we temporarily add a symmetric pair of occupied orbitals at degeneracy-free planes $\pm z_{0}$, and initially do not let them hop at all (completely isolated). This will introduce flat bands on those planes. Now let the added orbitals hybridize with other orbitals. Since accidental degeneracies away from the mirror planes are not protected, gaps will generally open up between the new and the old Wannier bands (the only exceptions to this rule are treated in the next paragraph). And since the added orbitals are topologically trivial, they have no effect on the MCNs, which can now be evaluated using the formalism of Sec. IV A for gapped spectra. Setting $\bar{C}_{\mathrm{A}}=\bar{C}_{\mathrm{B}}=0$ in Eqs. (33) and (34) therein, we obtain

$$
\mu_{\mathrm{G}}=\frac{1}{2}\left(W_{\mathrm{A}}+W_{\mathrm{B}}\right)
$$

and

$$
\mu_{\mathrm{X}}=\frac{1}{2}\left(W_{\mathrm{A}}-W_{\mathrm{B}}\right) .
$$

But since $W_{\mathrm{A}}$ and $W_{\mathrm{B}}$ cannot be affected by orbitals inserted far from the A and B planes, we conclude that Eqs. (42) and (43) can be directly applied to the original system with a gapless Wannier spectrum.

The above argument needs to be refined if the system is an axion-odd insulator that has, in addition to $M_{z}$ symmetry, one or more axion-odd symmetries that are $z$ preserving and symmorphic (e.g., spinful time reversal or vertical mirrors). The Wannier spectrum is then guaranteed to be gapless, with adjacent bands touching at an odd number of Dirac nodes [13]. The solution is to weakly break all such symmetries via some low-symmetry perturbation; the band connectivity then becomes "fragile," allowing gaps to open up once the added orbitals hybridize with the original ones [13,25]. The rest of the argument proceeds as before, again with the conclusion that Eqs. (42) and (43) can be directly applied to the original system with a gapless spectrum. This scenario is illustrated in Sec. VIC 2, where the orbital insertion itself acts as the symmetry-lowering perturbation.

To conclude, let us show that the relation (37) between the MCNs and the axion angle remains valid for gapless spectra. Equations (42) and (43) give $\mu_{\mathrm{G}}+\mu_{\mathrm{X}}=W_{\mathrm{A}}$, while $\theta$ is equal to the sum of Berry phases of vanishingly small loops around the nodes at A [13]. Since those Berry phases divided by $\pi$ are 
equal to the node winding numbers modulo 2 [26], Eq. (37) is immediately recovered.

\section{METHODS}

\section{A. Tight-binding, ab initio, and Wannier methods}

In this work, the formalism for evaluating MCNs in the HW representation is implemented in the tight-binding (TB) framework, using a modified version of the PYTHTB code [27]. Illustrative calculations are carried out for 2D and 3D models with mirror symmetry; some are simple toy models, while others are obtained from $a b$ initio calculations as described below. Each model is specified by providing the onsite energies, the hopping amplitudes, and the matrix elements of the position and mirror operators.

In the TB literature, it is common to assume that the position operator is represented by a diagonal matrix in the TB basis,

$$
\left\langle\varphi_{\mathbf{R} i}|\mathbf{r}| \varphi_{\mathbf{R}^{\prime} j}\right\rangle=\left(\mathbf{R}+\boldsymbol{\tau}_{i}\right) \delta_{\mathbf{R}, \mathbf{R}^{\prime}} \delta_{i j},
$$

where $\boldsymbol{\tau}_{i}$ is the location of the $i$ th basis orbital in the home cell $\mathbf{R}=\mathbf{0}$. This approximation is problematic for calculating the Wannier bands of unbuckled monolayers, since it forces all bands to lie flat on the $z=0$ plane: when all basis orbitals lie on the $z=0$ plane and all off-diagonal matrix elements $\left\langle\varphi_{\mathbf{R} i}|z| \varphi_{\mathbf{R}^{\prime} j}\right\rangle$ vanish, the matrix $Z_{\kappa}$ that is diagonalized to obtain the HW centers [see Eqs. (45) and (46)] is the null matrix.

To apply our formalism to flat monolayers, any flat Wannier bands that may be present must be robust and satisfy the uniform parity assumption, while all other bands must be dispersive. To ensure that this is so, one should retain some off-diagonal $z$ matrix elements. For models based on ab initio Wannier functions this occurs naturally, since the position matrix elements between the Wannier functions are explicitly calculated, and they are generally nonzero for nearby Wannier functions. In the case of toy models, one needs to assign nonzero values to some of the off-diagonal $z$ matrix elements under reasonable assumptions.

The material chosen for the ab initio calculations is SnTe, which we study as a flat monolayer in Sec. VI A and as a bulk phase in Sec. VIB. We first calculate the electronic structure from density-functional theory (DFT) using the GPAW code [28], and then use the WANNIER90 code [29] to construct welllocalized Wannier functions. Last, TB models are generated by tabulating the matrix elements of the Kohn-Sham Hamiltonian and of the position operator between those Wannier functions.

The self-consistent DFT calculations are performed without including spin-orbit coupling, which is added afterwards non-self-consistently [30]. We use the Perdew-BurkeErnzerhof exchange-correlation functional [31,32], and describe the valence-core interaction via the projector augmented wave method [33]. The valence states are expanded in a plane-wave basis with an energy cutoff of $600 \mathrm{eV}$, and the $\mathrm{BZ}$ is sampled on $\Gamma$-centered uniform grids containing $6 \times 6 \times 1$ and $6 \times 6 \times 6$ points for monolayer and bulk SnTe, respectively. The projector augmented wave setup includes the $4 d$ semicore states of $\mathrm{Sn}$ in addition to the $5 s$ and $5 p$ states of $\mathrm{Sn}$ and Te, yielding a total of 20 valence electrons for each
SnTe formula unit (one per cell for the monolayer, and two for the bulk).

For each formula unit, we construct 16 spinor Wannier functions of $s$ and $p$ character spanning the upper-valence and low-lying conduction band states. The Sn $4 d$ states, which give rise to flat bands lying $22 \mathrm{eV}$ below the Fermi level, are excluded from the Wannier construction.

As a first step towards obtaining well-localized Wannier functions, we extract from the space of $a b$ initio Bloch eigenstates at each grid point $\mathbf{k}$ an $N$-dimensional subspace with the desired orbital character $(N=16$ for the monolayer, and $N=32$ for the bulk). This is achieved via the "band disentanglement" procedure of Ref. [34], which involves specifying two energy windows, known as the inner and the outer window, and a set of trial orbitals. The outer window encloses all the valence bands except for the $4 d$ semicore states, as well as all the low-lying conduction states of $5 s$ and $5 p$ character. To ensure that the valence states are exactly preserved in the disentangled subspace, we "freeze" them inside an inner window. An initial guess for the target subspace is obtained by projecting atom-centered $s$ and $p$ trial orbitals onto the outerwindow states. This is followed by an iterative procedure that yields an optimally smooth disentangled subspace across the BZ [34].

Having extracted a suitable Bloch subspace, we proceed to construct well-localized $s$ - and $p$-like Wannier functions spanning that subspace. This is done by projecting onto it the same $s$ and $p$ trial orbitals that were used in the disentanglement step, and then orthogonalizing the resulting orbitals via the Löwdin scheme [18]. This one-shot procedure, without additional maximal-localization steps [18], ensures that the Wannier functions retain the orbital character of the trial orbitals.

To assess the quality of the Wannier basis we calculate the energy bands from the Hamiltonian matrix elements in that basis [34], and find that they are in excellent agreement with the $a b$ initio bands obtained using the GPAW code [35].

In addition to the Hamiltonian and position matrix elements, we also require the matrix elements of the mirror operator $M_{z}$ in the Wannier basis. These are needed to determine the winding numbers of the nodal touchings between Wannier bands on the mirror planes (see Sec. V C), as well as the mirror parities $p_{\mathrm{A}}$ and $p_{\mathrm{B}}$ of the flat-band states. To set up the matrix representation of $M_{z}$, we assume that the Wannier functions transform under $M_{z}$ in the same way as pure $s$ and $p$ orbitals. We find that the eigenstates of the Wannier Hamiltonian on the mirror-invariant BZ planes are, to a good approximation, eigenstates of this approximate $M_{z}$ operator, which validates that assumption.

\section{B. Construction of hybrid Wannier functions and Wannier bands}

Formally, maximally localized HW functions satisfy the eigenvalue equation (12). For a 2D or quasi-2D system extended along $x$ and $y$, the matrix elements of the $z$ operator appearing in that equation are well defined. It is therefore straightforward to set up the matrix

$$
Z_{m n \mathbf{k}}=\left\langle\psi_{m \mathbf{k}}|z| \psi_{n \mathbf{k}}\right\rangle
$$


where $\mathbf{k}=\left(k_{x}, k_{y}\right)$ and $m$ and $n$ run over the $J$ occupied energy bands, and to diagonalize it,

$$
\left[U_{\mathbf{k}}^{\dagger} Z_{\mathbf{k}} U_{\mathbf{k}}\right]_{m n}=z_{m \mathbf{k}} \delta_{m n} .
$$

The eigenvalues are the HW centers, and from the eigenvectors (the columns of the $U_{\mathbf{k}}$ matrix) we can construct the maximally localized $\mathrm{HW}$ functions according to

$$
\left|h_{n \mathbf{k}}\right\rangle=\sum_{m} e^{-i \mathbf{k} \cdot \mathbf{r}}\left|\psi_{m \mathbf{k}}\right\rangle U_{m n \mathbf{k}}
$$

where the phase factor has been included to render them inplane periodic.

For bulk systems, which are extended in all directions including the Wannierization direction $z$, the above procedure fails because the matrix elements in Eq. (45) become ill defined. In such cases, it is still possible to construct maximally localized HW functions by working in reciprocal space. We now write $\mathbf{k}=\left(\boldsymbol{\kappa}, k_{z}\right)$, and choose a uniform grid; for each point $\boldsymbol{\kappa}$ in the projected 2D BZ, the problem reduces to the construction of $1 \mathrm{D}$ maximally localized Wannier functions along $z$. The procedure is detailed in Refs. [5,18]. Briefly, the first step is to establish a "twisted parallel transport gauge" for the valence Bloch states along the string of $k_{z}$ points at each $\kappa$, obtaining as a byproduct the HW centers $z_{l n \kappa}$. The maximally localized HW functions $\left|h_{l n \kappa}\right\rangle$ are then constructed in this gauge using Eq. (11), with the integral over $k_{z}$ replaced by a summation over the string of $k_{z}$ points.

\section{Winding number of a point node of order $N$ 1. Definition}

Earlier we defined the winding number of a point node where two Wannier bands meet on a mirror plane. Since there are situations where $N>1$ pairs of bands meet at a node, we need to generalize that definition to handle such "higherorder" nodes.

Given a point node $\boldsymbol{\kappa}_{j}$ of order $N \geqslant 1$, we introduce the $2 N \times 2 N$ matrix representation of $M_{z}$ at a nearby point $\kappa$,

$$
\mathcal{M}_{m n \kappa}^{z}=\left\langle h_{m \kappa}\left|M_{z}\right| h_{n \kappa}\right\rangle \text {. }
$$

Here, $m$ and $n$ run over the $2 N$ Wannier bands that meet at $\boldsymbol{\kappa}_{j}$. By diagonalizing $\mathcal{M}_{\kappa}^{z}$ and then transforming the $\left|h_{n \kappa}\right\rangle$ states accordingly [see Eqs. (46) and (47)], we obtain a new set of $2 N$ states $\left|\tilde{h}_{n \kappa}\right\rangle$. Like the original ones they are cell-periodic in plane and localized along $z$, but they have definite mirror parities. We choose the first $N$ to be even under $M_{z}$, and denote them as $\left|\tilde{h}_{l \kappa}^{+}\right\rangle$; the remaining $N$ are odd under $M_{z}$, and we denote them as $\left|\tilde{h}_{l \kappa}^{-}\right\rangle$. In both cases, $l$ goes from 1 to $N$. The matrix representation of $z$ in the new basis takes the form of Eq. (23), where $f_{\kappa}$ is the $N \times N$ matrix with elements

$$
f_{l l^{\prime} \kappa}=\left\langle\tilde{h}_{l \kappa}^{+}|z| \tilde{h}_{l^{\prime} \kappa}^{-}\right\rangle .
$$

Letting

$$
\gamma_{\kappa}=\arg \left(\operatorname{det} f_{\kappa}\right),
$$

the winding number can be evaluated from Eq. (28) irrespective of the order $N$ of the node.
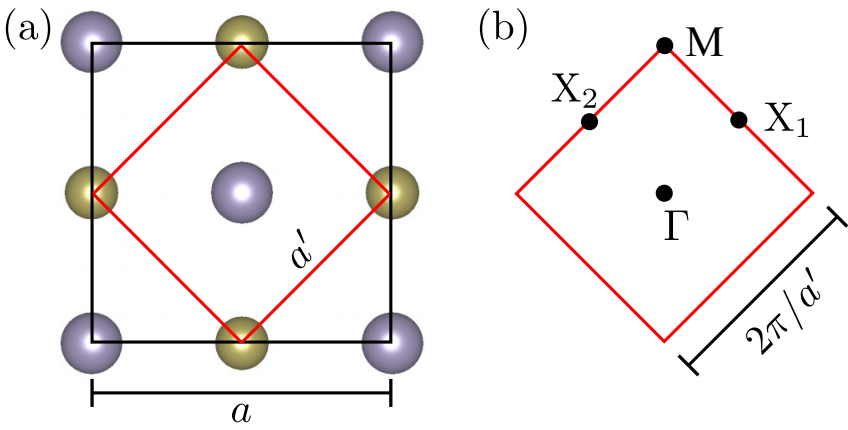

FIG. 2. (a) Atomic structure of monolayer SnTe. The black square is the conventional unit cell with lattice constant $a$, and the red square is the primitive cell with lattice constant $a^{\prime}=a / \sqrt{2}$. (b) Brillouin zone and high-symmetry points.

\section{Numerical evaluation}

Suppose a single pair of Wannier bands meet at a point node $\boldsymbol{\kappa}_{j}$. To evaluate the winding number (28), the phase $\gamma_{\kappa}$ must be smooth on $c_{j}$. In practice, we establish a smooth gauge for the states $\left|\tilde{h}_{\kappa}^{ \pm}\right\rangle$as follows. We pick a representation of the two states at a reference point $\boldsymbol{\kappa}_{j}^{\prime}$ in the vicinity of the node. Then at any point $\boldsymbol{\kappa}_{j}^{\prime}+\Delta \boldsymbol{\kappa}$ on the circle $c_{j}$ we choose the gauge by enforcing maximal phase alignment with the states at $\kappa_{j}^{\prime}$, i.e., by requiring that the overlaps $\left\langle\tilde{h}_{\kappa_{j}^{\prime}}^{+} \tilde{h}_{\kappa_{j}^{\prime}+\Delta \kappa}^{+}\right\rangle$ and $\left\langle\tilde{h}_{\kappa_{j}^{\prime}}^{-} \mid \tilde{h}_{\kappa_{j}^{\prime}+\Delta \kappa}^{-}\right\rangle$are real and positive. In other words, we carry out a one-step parallel transport from $\boldsymbol{\kappa}_{j}^{\prime}$ to each circumference point.

If several pairs of bands meet at a node, the strategy is basically the same. The only difference is that one must now use the multiband version of the parallel-transport procedure $[5,18]$.

\section{NUMERICAL RESULTS}

In this section, we use our formalism to calculate the MCNs of three different systems. The first is an unbuckled monolayer of $\mathrm{SnTe}$, a topological crystalline insulator protected by reflection symmetry about its plane. The second is rock salt SnTe, a 3D topological crystalline insulator protected by a type- 2 mirror. Our last example is a 3D toy model based on a modified Dirac equation. It is both a strong topological insulator protected by time-reversal symmetry, and a topological crystalline insulator with a type-1 mirror. In the first example the Wannier spectrum is trivially gapped, while in the other two it is gapless.

\section{A. Unbuckled monolayer of SnTe}

The structure we consider is shown in Fig. 2(a). It consist of a single unbuckled layer of $\mathrm{Sn}$ and Te atoms arranged in a checkerboard pattern, which can be viewed as a single (001) layer of the bulk rock salt structure.

DFT calculations reveal that the system with an optimized lattice constant of $a=6.16 \AA$ is situated $0.4 \mathrm{eV}$ above the convex hull and is dynamically unstable [36], and that a buckled structure that breaks mirror symmetry is energetically favored [37]. These results imply that a flat SnTe monolayer 

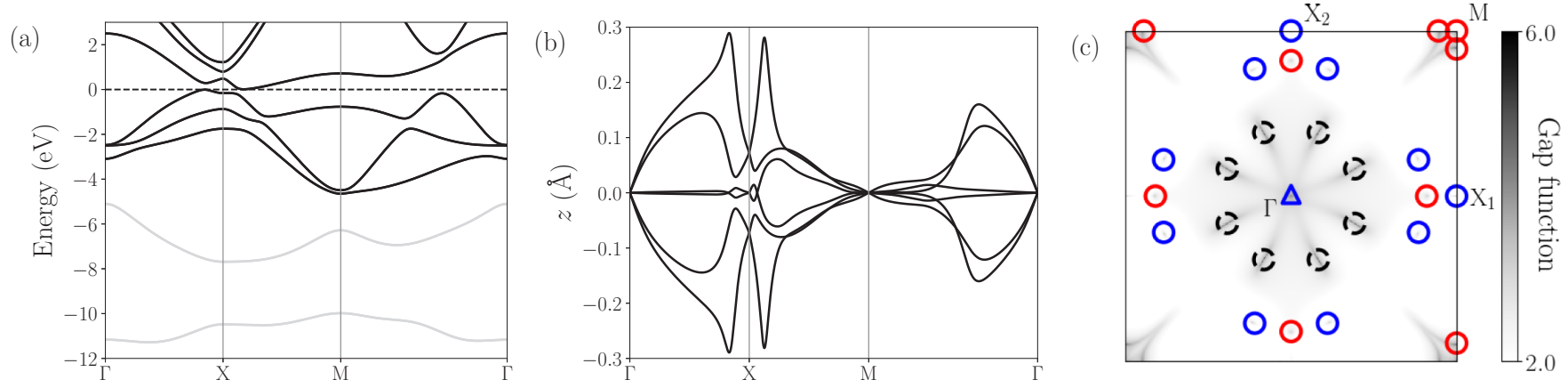

FIG. 3. (a) Energy bands of monolayer SnTe, with the $s$-type lower valence bands that are excluded from the Wannierization shown in gray. All bands are doubly degenerate, and the Fermi level is indicated by the dashed line. (b) Wannier bands obtained from the Bloch states in the six p-type upper valence bands. (c) Heat map plot of the gap function of Eq. (52) for the central pair of Wannier bands, where zero-gap points (nodal points) appear as dark spots. Those with winding numbers $W_{j}= \pm 1$ are indicated by red or blue circles, while the one with $W_{j}=-3$ at the $\Gamma$ point is indicated by a blue triangle. Dashed circles denote pairs of nearby nodes with equal and opposite winding numbers. When a node falls on the BZ boundary, only one of the periodic images is shown.

is not likely to be experimentally relevant. This system is nevertheless ideally suited for illustrating our methodology, since it has reflection symmetry about its own plane and the associated $\mathrm{MCN}$ is nonzero [38].

We carry out calculations using the primitive cell containing one formula unit. The Wannier-interpolated energy bands are shown in Fig. 3(a), where all bands are doubly degenerate due to time-reversal and inversion symmetry. There is a robust inverted gap $(0.3 \mathrm{eV})$ at the $\mathrm{X}$ point, and a tiny indirect gap $(0.17 \mathrm{meV})$ around the $\mathrm{X}$ point; when the lattice expands the indirect gap increases, and when it shrinks the system turns into a band overlap semimetal [37,38]. The lowest four valence bands are predominantly $s$-type, and the remaining six (plotted in red) are predominantly $p$-type.

Figure 3(b) shows the Wannier bands calculated from the Bloch states in the $p$-type upper valence bands. The spectrum consists of three mirror-symmetric band pairs that touch on the A plane $z=0$ at isolated points in the 2D BZ. There are no flat bands on that plane, as expected from the presence of time-reversal symmetry (Sec. III B 3). Equation (40) therefore reduces to

$$
\mu_{2 \mathrm{D}}=\frac{1}{2} W_{\mathrm{A}},
$$

and the MCN can be determined by evaluating the winding numbers of the nodal points on the A plane.

To locate those nodal points, we plot in Fig. 3(c) the "gap function"

$$
g_{\mathbf{k}}=-\log \left(\Delta z_{\mathbf{k}} / c\right),
$$

where $\Delta z(\mathbf{k})$ is the separation between the central pair of bands. Regions with a small gap appear in dark gray, and nodal points as dark spots. The positions and winding numbers of all the nodal points are indicated in the figure, where we have included only one of the periodic images when a node falls on the BZ boundary. At $\Gamma$ and $\mathrm{M}$ there are nodes where three pairs of Wannier bands touch, with winding numbers $W_{j}=-3$ and $W_{j}=+1$, respectively. All other nodes on the $z=0$ plane are simple Dirac nodes where only the two central bands meet, and they have $W_{j}= \pm 1$. Adding up the winding numbers of the 36 nodal points in the $\mathrm{BZ}$ we obtain $W_{\mathrm{A}}=-4$, and from Eq. (51) we conclude that the group of six $p$-type valence bands has a MCN of -2 .

We repeat the calculation for the four $s$-type lower valence bands, and find that their net winding number vanishes. The net $\mathrm{MCN}$ of the occupied states is therefore $\mu_{2 \mathrm{D}}=-2$, with the nontrivial topology coming from the $p$ states. This result agrees with the value $\left|\mu_{2 \mathrm{D}}\right|=2$ inferred from a $k \cdot p$ analysis of the simultaneous band inversions at the two $\mathrm{X}$ points in the $\mathrm{BZ}[17,38]$.

\section{B. Bulk SnTe}

Bulk SnTe, which crystallizes in the rock salt structure, is known both from theory [16] and experiment [39] to be a topological crystalline insulator. The symmetry protecting its nontrivial band topology is reflection about the $\{110\}$ family of planes. [Instead, the (001) mirror symmetry responsible for the topological state of the monolayer is topologically trivial in the bulk crystal.]

The lattice is face-centered cubic lattice, so that the shortest lattice vector perpendicular to the (110) planes is $\mathbf{a}_{3}=a \hat{\mathbf{x}} / 2+$ $a \hat{\mathbf{y}} / 2$. Since its length is twice the separation between adjacent planes, the (110) mirror operation is of type 2, as is typical of centered lattices (see Fig. 1).

For our simulations we pick a tetragonal cell subtended by $\mathbf{a}_{1}=-a \hat{\mathbf{x}} / 2+a \hat{\mathbf{y}} / 2, \mathbf{a}_{2}=a \hat{\mathbf{z}}$, and $\mathbf{a}_{3}$, and reorient the axes such that those vectors point along $\hat{\mathbf{x}}, \hat{\mathbf{y}}$, and $\hat{\mathbf{z}}$, respectively. In this new frame, the (110) mirror operation of interest becomes $M_{z}$. The simulation cell with two formula units is shown in Fig. 4(a) and the associated BZ in Fig. 4(b).

In Fig. 5(a) we present the energy bands calculated along the high-symmetry lines of the folded BZ. The nontrivial topology arises from simultaneous band inversions at the two $\mathrm{L}$ points in the unfolded BZ [16], which map onto the two $\mathrm{R}$ points in Fig. 4(b). The inverted band gap at $\mathrm{R}$ and the global indirect band gap amount to 0.3 and $0.1 \mathrm{eV}$, respectively.

From the full set of valence band states, we construct HW functions localized along $z$. The Wannier spectrum is shown in Fig. 5(b). Its periodicity is $c / 2$ because the cell is doubled along $z$, and only one period is shown. The spectrum is gapless, with two pairs of bands crossing in opposite directions, 
(a)

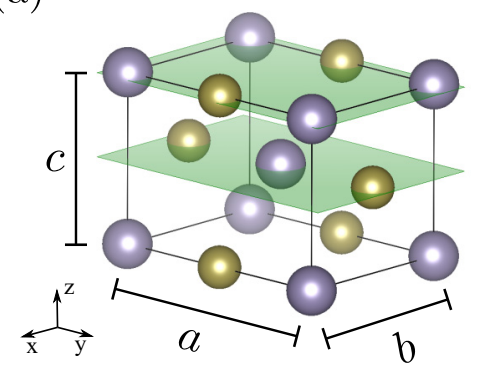

FIG. 4. (a) Rock salt structure of bulk SnTe in a tetragonal conventional cell. $a$ is the lattice constant of the conventional cubic cell, and $b=c=a / \sqrt{2}$. Green planes are equivalent mirror planes. (b) Brillouin zone associated with the tetragonal cell, with its highsymmetry points indicated in red and the unique $M_{z}$-invariant plane in green. The projected 2D Brillouin zone with its high-symmetry points is shown on top.

between $\overline{\mathrm{X}}$ and $\bar{\Gamma}$, the gap centered at $z=c / 4$ (only one of the two crossings is shown). This spectral flow arises from the nonzero MCN associated with $M_{y}$ symmetry (equivalent to $M_{z}$ ), which leaves invariant the $\mathrm{BZ}$ plane containing the

(b)

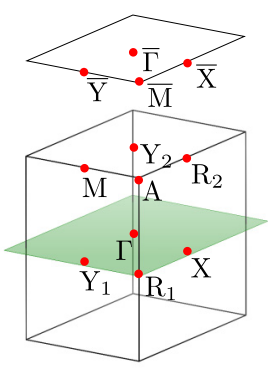

$\Gamma, \mathrm{X}, \mathrm{R}_{2}$, and $\mathrm{Y}_{2}$ points. For a discussion of such "in-plane" Wannier flow associated with a nonzero MCN, see Ref. [40].

Since $M_{z}$ is a type- 2 mirror, we evaluate its unique MCN using Eq. (38). And since the Wannier spectrum is gapless, and hence devoid of flat bands, we set $\bar{C}_{\mathrm{A}}=0$ in that equation to obtain

$$
\mu_{\mathrm{G}}=W_{\mathrm{A}},
$$

which says that the MCN equals the sum of the winding numbers of all the point nodes on the $z=0$ plane.

As indicated in Fig. 5(d), there are 16 independent point nodes in total on that plane, all of them simple nodes where only two bands meet. Seven have winding numbers +1 and the other nine have winding numbers -1 , yielding $\mu_{\mathrm{G}}=-2$ for the MCN. This value is in agreement with that originally obtained in Ref. [16] from a $k \cdot p$ analysis of the band inversions. Using Eq. (39), we confirm that the system is axion-trivial.

\section{Modified Dirac model on a cubic lattice}

In this section we study a 3D toy model constructed by first modifying the free Dirac equation to enable topological phases for certain parameter values, and then placing it on a cubic lattice. The $4 \times 4$ Hamiltonian matrix in reciprocal space reads $[41,42]$

$$
H(\mathbf{k})=\left(\begin{array}{cccc}
m-2 M K(\mathbf{k}) & 0 & c \sin k_{z} & c\left(\sin k_{x}-i \sin k_{y}\right) \\
0 & m-2 M K(\mathbf{k}) & c\left(\sin k_{x}+i \sin k_{y}\right) & -c \sin k_{z} \\
c \sin k_{z} & c\left(\sin k_{x}-i \sin k_{y}\right) & -m+2 M K(\mathbf{k}) & 0 \\
c\left(\sin k_{x}+i \sin k_{y}\right) & -c \sin k_{z} & 0 & -m+2 M K(\mathbf{k})
\end{array}\right),
$$

where $K(\mathbf{k})=3-\cos k_{x}-\cos k_{y}-\cos k_{z}$, and $c, m$, and $M$ are dimensionless parameters inherited from the original isotropic modified Dirac equation [41] by setting the rest mass $m_{0} c^{2}$ to be the energy scale of the model [42].

The topological phase diagram of the half-filled model is shown in Fig. 6 for $c=1.0$. The system is gapped except on the $m=0,4 M, 8 M, 12 M$ lines, where the gap closes at $\Gamma=$ $(0,0,0), X=(\pi, 0,0), M=(\pi, \pi, 0)$, and $A=(\pi, \pi, \pi)$, respectively. As shown in Appendix C, those metallic lines separate axion-trivial from axion-odd insulating phases.

The axion angle is quantized by several axion-odd symmetries. Some are $z$-reversing (inversion and horizontal mirror $M_{z}$ ), and others are $z$-preserving (spinful time reversal and vertical mirrors). As $M_{z}$ is a type- 1 mirror, it protects two MCNs that are related to the axion angle by Eq. (37).

\section{Axion-odd phase with protected Wannier flow}

For our numerical tests we set $c=m=1.0$ and $M=0.5$ to put the model in the axion-odd phase. The energy band structure is shown in Fig. 7(a). The bands are pairwise degenerate due to the presence of time-reversal and inversion symmetry, with a finite gap between the two pairs over the entire BZ. The Fermi level is placed at midgap.

Since the system is axion-odd and has $z$-preserving axionodd symmetries, the connectivity (or "flow") of the Wannier bands is topologically protected [13]. In particular, spinful time-reversal symmetry requires that the two bands per vertical cell are glued together as follows: one band touches the band above at one of the four time-reversal invariant momenta (TRIM), and it touches the periodic image below at the other three. As for the $z$-reversing axion-odd symmetries, the effect of $M_{z}$ is to pin the up-touching to one of the mirror planes and the three down-touchings to the other, while inversion further constrains the four touchings to occur at TRIM on those planes, as already mandated by time reversal.

The pattern of band touchings described above is confirmed by Fig. 7(b), where we plot the Wannier bands. They were obtained by placing at the origin the four basis orbitals that belong to the home unit cell, and making the diagonal approximation of Eq. (44) for the position matrix. There is one band touching at $\bar{\Gamma}$ on the B plane, and three more on the A plane: one at $\overline{\mathrm{M}}$, and the others at the two $\overline{\mathrm{X}}$ points.

Since the Wannier spectrum is gapless, the MCNs $\mu_{\mathrm{G}}$ and $\mu_{\mathrm{X}}$ are given respectively by the half-sum and the halfdifference of the net winding numbers on the $\mathrm{A}$ and $\mathrm{B}$ planes [Eqs. (42) and (43)]. As indicated in the gap-function plots of Figs. 7(c) and 7(d), the three nodes at $\mathrm{A}$ give $W_{\mathrm{A}}=-1$ and the single node at $\mathrm{B}$ gives $W_{\mathrm{B}}=-1$, so that $\mu_{\mathrm{G}}=-1$ and $\mu_{\mathrm{X}}=0$. Note that $\mu_{\mathrm{G}}+\mu_{\mathrm{X}}$ is an odd number, as required by Eq. (37) for an axion-odd system. 

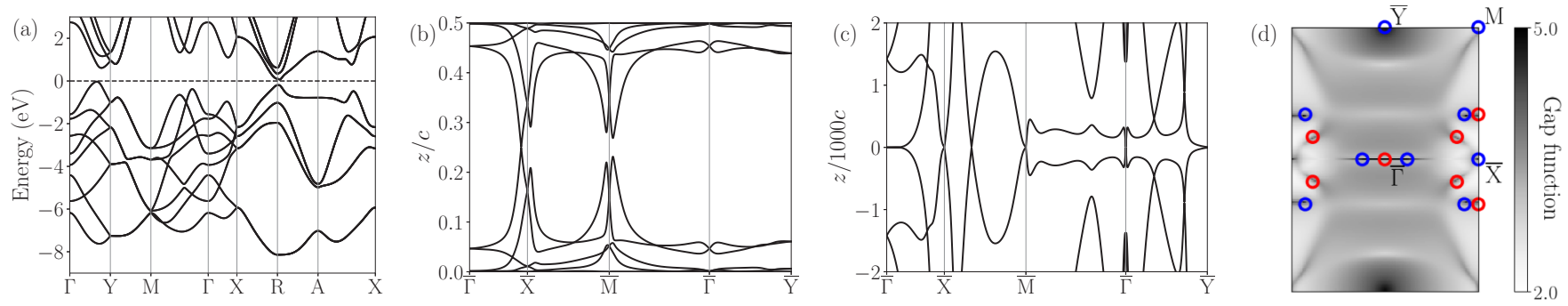

FIG. 5. (a) Energy bands of bulk SnTe along high-symmetry lines of the folded tetragonal BZ. The Fermi level is indicated by the dashed line. (b) Wannier band structure obtained from the full set of valence states. (c) Detail of the Wannier bands around the $z=0$ mirror plane. (d) Heat map plot of the gap function of Eq. (52) for the central pair of Wannier bands around $z=0$, with the nodal points color coded as in Fig. 3(c).

\section{Axion-odd phase with fragile Wannier flow}

If the $z$-preserving axion-odd symmetries of the model (time reversal and vertical mirrors) are weakly broken, the system will remain in an axion-odd phase protected by $M_{z}$ and inversion. But since these are $z$-reversing operations, the Wannier spectrum is no longer topologically required to be gapless. The Wannier flow is protected only in a "fragile" sense, and it can be destroyed, while preserving $M_{z}$, by adding some weakly coupled trivial bands to the valence manifold $[13,25]$. Below we carry out this procedure in two different ways, and confirm that the MCNs remain the same as in the original model.

a. Insertion of a symmetric pair of occupied orbitals. Here we implement the strategy outlined in Sec. IV B. We insert in the unit cell two more orbitals, denoted as $|5\rangle$ and $|6\rangle$, that have opposite spins and the same on-site energy $E=-4.0$. To break time reversal and the vertical mirrors while preserving $M_{z}$ and inversion, we place the spin-up orbital $|5\rangle$ at $(x, y, z)=(0.0,0.0,0.2 c)$, and the spin-down orbital $|6\rangle$ at $(x, y, z)=(0.0,0.0,-0.2 c)$, keeping the original orbitals $|1\rangle$ to $|4\rangle$ at the origin. Finally, we couple the new orbitals to the old via the matrix elements $\langle 5|H| 1\rangle=\langle 6|H| 2\rangle=0.5$. The resulting model retains the $M_{z}$ and inversion symmetries of the original model, and it breaks the time-reversal and vertical mirror symmetries in the $Z$ matrix of Eq. (45) (but not in the Hamiltonian).

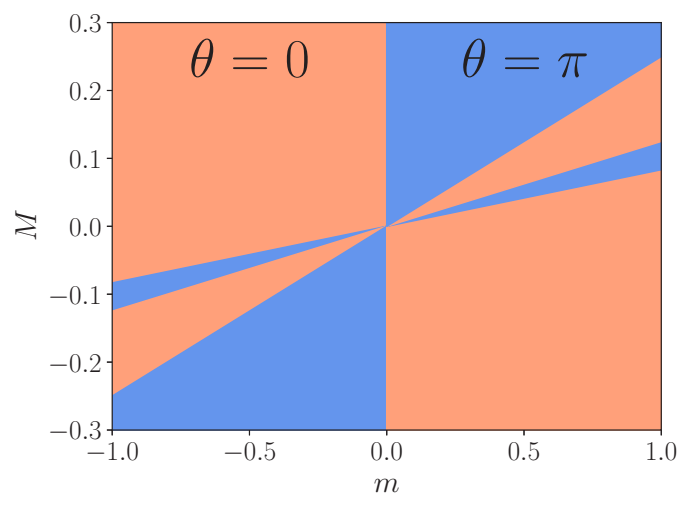

FIG. 6. Topological phase diagram of the model of Eq. (54) for $c=1.0$. Orange and blue regions denote axion-even $(\theta=0)$ and axion-odd $(\theta=\pi)$ phases, respectively.
The energy and Wannier band structures are plotted in Figs. 8(a) and 8(b). Because the Hamiltonian has both inversion and time-reversal symmetry, the energy bands remain doubly degenerate as in Fig. 7(a). The breaking of the $z$-preserving symmetries in the $Z$ matrix is reflected in the Wannier spectrum which is no longer connected as in Fig. 7(b), with small gaps opening up near $z= \pm 0.2 c$. The node at $\bar{\Gamma}$ on the $\mathrm{B}$ plane and those at $\overline{\mathrm{X}}_{1}, \overline{\mathrm{X}}_{2}$, and $\overline{\mathrm{M}}$ on the A plane remain intact, protected by $M_{z}$ and inversion. Their winding numbers are also unchanged, leading to the same MCNs as in the original model.

$b$. Insertion of a single occupied orbital at $z=0$. An alternative way of opening up a gap in the Wannier spectrum is to insert a flat band on a mirror plane. To illustrate this procedure, we add at the origin a single spin-up orbital $|5\rangle$ with on-site energy $E=-4.0$ and odd parity about that plane, and couple it to the model via $\langle 5|H| 1\rangle=\langle 5|H| 4\rangle=2.0$. Because the orbital is spin-polarized, it breaks time reversal; and because the spin points in the vertical direction, it also breaks all vertical mirrors while preserving $M_{z}$. In addition, the coupling terms break inversion symmetry, leaving $M_{z}$ as the only axion-odd symmetry. The energy bands of the modified model
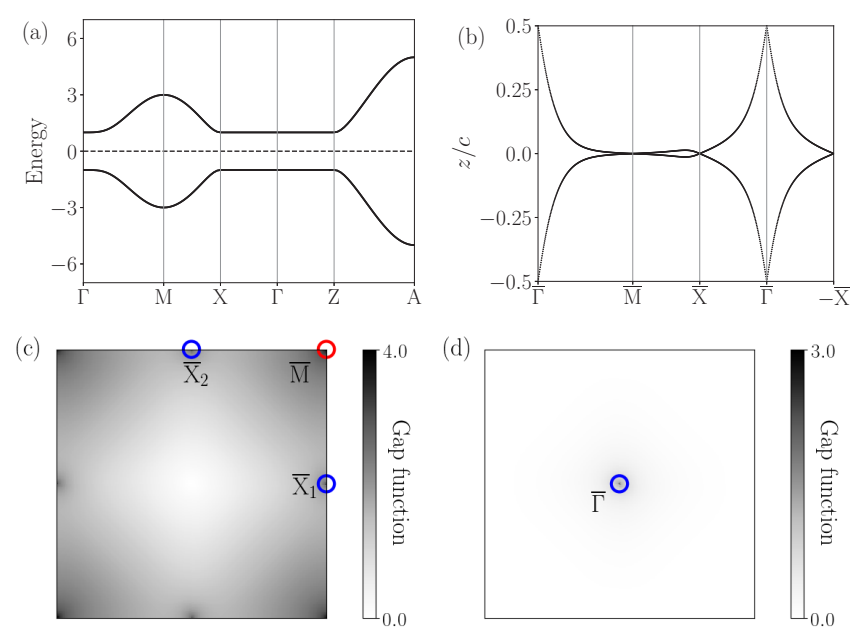

FIG. 7. (a) Energy bands of the model described by Eq. (54) with $c=m=1.0$ and $M=0.5$. The bands are doubly degenerate, and the Fermi level (dashed line) has been placed at midgap. (b) Wannier band structure obtained from the valence states. (c) and (d) Heat map plots of the gap function of Eq. (52) about the $z=0$ and $z=c / 2$ planes, respectively, with the nodal points color coded as in Fig. 3(c). 

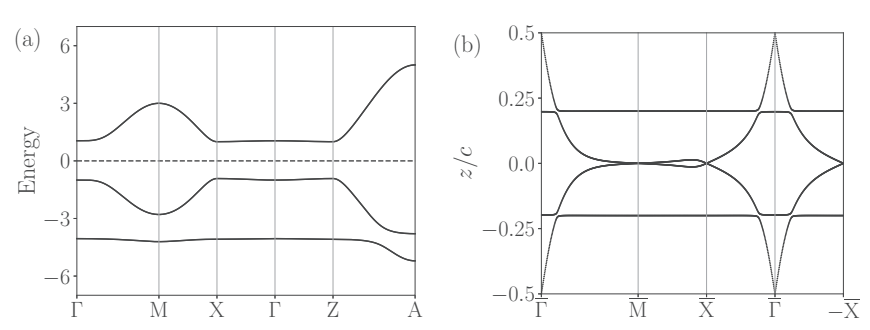

FIG. 8. (a) Energy bands of the same model as in Fig. 7, after adding an extra pair of occupied orbitals with $E=-4.0$ at $z=$ $\pm 0.2 c$ and coupling them to the other orbitals. The bands are doubly degenerate, and the Fermi level (dashed line) has been placed at midgap. (b) Wannier band structure obtained from the valence states, with small gaps around $z= \pm 0.2 c$ due to the added orbitals.

are shown in Fig. 9(a). A new band has appeared below the other four, so that there are now three valence bands in total, leading to three Wannier bands.

The added orbital, which belongs to the $\mathrm{A}^{+}$class in Table I, generates an extra even-parity state at both $\mathrm{G}$ and $\mathrm{X}$. This creates an imbalance $\Delta N_{\mathrm{G}}=\Delta N_{\mathrm{X}}=1$ between even- and odd-parity states on the two mirror-invariant BZ planes, which according to Eq. (20) results in a flat band at A. We emphasize that this extra band remains flat even after the added orbital is coupled to the model, as long as the coupling terms respect $M_{z}$ symmetry. As already mentioned, those terms are chosen to break inversion symmetry. This is needed to ensure that the three point nodes on the A plane are repelled by the flat band in the manner described in Sec. III B 2, since inversion symmetry would otherwise protect them.

The resulting Wannier bands are displayed in the upper panel of Fig. 9(b); because of the lowered symmetry, the node at $z=c / 2$ is no longer pinned to $\bar{\Gamma}$ as in Fig. 7(b). The lower panel reveals a perfectly flat band at $z=0$, well separated from a pair of dispersive bands whose three touchings on the $z=0$ plane in Fig. 7(c) have been gapped out. Under these circumstances, Eqs. (33) and (34) for the MCNs reduce to

$$
\mu_{\mathrm{G}}=\frac{1}{2}\left(p_{\mathrm{A}} \bar{C}_{\mathrm{A}}+W_{\mathrm{B}}\right)
$$

and

$$
\mu_{\mathrm{X}}=\frac{1}{2}\left(p_{\mathrm{A}} \bar{C}_{\mathrm{A}}-W_{\mathrm{B}}\right)
$$
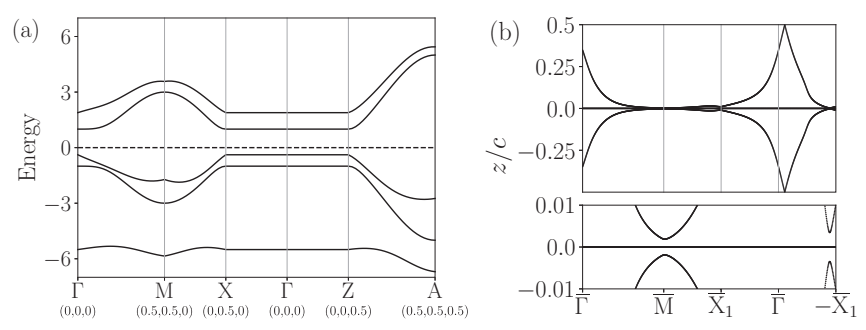

FIG. 9. (a) Energy bands of the same model as in Fig. 7, after adding an extra occupied orbital at $z=0$ and coupling it to the other orbitals. The Fermi level (dashed line) has been placed in the gap. (b) Wannier band structure obtained from the valence states. The added orbital generates a flat band at $z=0$, which repels the nodal points on that plane (lower panel).
The single node at $\mathrm{B}$ has the same winding number $W_{\mathrm{B}}=$ -1 as in the original model, while the net winding number $W_{\mathrm{A}}=-1$ of the gapped-out nodes at $\mathrm{A}$ has been transferred to the index $p_{\mathrm{A}} \bar{C}_{\mathrm{A}}$ of the flat band $\left(p_{\mathrm{A}}=-1\right.$, and $\left.\bar{C}_{\mathrm{A}}=+1\right)$. Overall, the MCNs remain unchanged.

\section{SUMMARY}

In summary, we have investigated the topological properties of mirror-symmetric insulating crystals from the viewpoint of $\mathrm{HW}$ functions localized along the direction orthogonal to the mirror plane. We first clarified the generic behaviors of the associated Wannier bands, and then derived a set of rules for deducing the MCNs. To validate and illustrate the formalism, we applied it to SnTe in the monolayer and bulk forms, and to a toy model of an axion-odd insulator.

In the HW representation, the MCNs are expressed in terms of a set of integer-valued properties of the Wannier bands on the mirror planes: the Chern numbers and mirror parities of flat bands lying on those planes, and the winding numbers of the touching points on those planes between symmetric pairs of dispersive bands. One advantage of this representation is that it reveals the relation between the MCNs and the axion $\mathbb{Z}_{2}$ index from purely bulk considerations. That relation is far from obvious in the standard Bloch representation, and previously it had been obtained only via an indirect argument involving surface states.

In some cases the axion $\mathbb{Z}_{2}$ index can be determined by visual inspection of the Wannier band structure, e.g., by counting the number of nodal points between certain bands [13]. We have found that mere visual inspection does not suffice for obtaining the MCNs since it does not reveal, for example, the relative signs of the winding numbers of different nodes.

Interestingly, in certain cases where flat Wannier bands are present the magnitudes of the MCN can be determined without having to divide the occupied manifold into two mirror sectors. This follows from the uniform-parity assumption for the flat bands, which has no counterpart in the Bloch representation. Since the determination of the mirror parities is the most cumbersome step in the calculation of MCNs, this feature of the HW formalism could lead to a more automated algorithm for computing MCNs. Even without such further developments, the formalism has already proven useful for discussing the topological classification of mirror-symmetric insulators.

\section{ACKNOWLEDGMENTS}

Work by T.R. was supported by the Deutsche Forschungsgemeinschaft Grant No. Ra 3025/1-1 from the Deutsche Forschungsgemeinschaft. Work by D.V. was supported by National Science Foundation Grant DMR-1954856. Work by I.S. was supported by Grant No. FIS2016-77188-P from the Spanish Ministerio de Economía y Competitividad. 


\section{APPENDIX A: DERIVATION OF EQS. (20)-(22)}

According to Table I, the numbers of occupied states with each mirror parity at $\mathrm{G}$ and $\mathrm{X}$ are

$$
\begin{aligned}
& N_{\mathrm{G}^{ \pm}}=\bar{N}_{\mathrm{A}^{ \pm}}+\bar{N}_{\mathrm{B}^{ \pm}}+\frac{1}{2} \tilde{N}, \\
& N_{\mathrm{X}^{ \pm}}=\bar{N}_{\mathrm{A}^{ \pm}}+\bar{N}_{\mathrm{B}^{\mp}}+\frac{1}{2} \tilde{N},
\end{aligned}
$$

where $\widetilde{N}=\widetilde{N}_{\mathrm{A}}+\widetilde{N}_{\mathrm{B}}+\widetilde{N}_{\mathrm{UC}}$ is the total number of dispersive Wannier bands per cell. Letting $\Delta N_{\mathrm{G}}=N_{\mathrm{G}^{+}}-N_{\mathrm{G}^{-}}$and $\Delta \bar{N}_{\mathrm{A}}=\bar{N}_{\mathrm{A}^{+}}-\bar{N}_{\mathrm{A}^{-}}$, and defining $\Delta N_{\mathrm{X}}$ and $\Delta N_{\mathrm{B}}$ in the same way, we find

$$
\begin{aligned}
\Delta \bar{N}_{\mathrm{A}} & =\frac{1}{2}\left(\Delta N_{\mathrm{G}}+\Delta N_{\mathrm{X}}\right), \\
\Delta \bar{N}_{\mathrm{B}} & =\frac{1}{2}\left(\Delta N_{\mathrm{G}}-\Delta N_{\mathrm{X}}\right) .
\end{aligned}
$$

Under the uniform parity assumption $\left|\Delta \bar{N}_{\mathrm{A}}\right|=\bar{N}_{\mathrm{A}}$ and $\left|\Delta \bar{N}_{\mathrm{B}}\right|=\bar{N}_{\mathrm{B}}$, resulting in Eqs. (20) and (21). In the case of a type-2 mirror $\mathrm{A}$ and $\mathrm{B}$ are equivalent, and from Eq. (A1a) $\Delta \bar{N}_{\mathrm{A}}+\Delta \bar{N}_{\mathrm{B}}=\Delta N_{\mathrm{G}}$. Hence $\Delta \bar{N}_{\mathrm{A}}=\Delta \bar{N}_{\mathrm{B}}=\Delta N_{\mathrm{G}} / 2$, yielding Eq. (22) under the same assumption.

\section{APPENDIX B: DERIVATION OF EQ. (27)}

Let us prove Eq. (27) for the case of a single pair of dispersive Wannier bands connected by point nodes on the A plane. In this case the matrix $f_{\kappa}$ of Eq. (49) reduces to the scalar

$$
f_{\kappa} \equiv\left\langle\widetilde{h}_{\kappa}^{+}|z| \widetilde{h}_{\kappa}^{-}\right\rangle=\left|f_{\kappa}\right| e^{i \gamma_{\kappa}},
$$

where $\left|\tilde{h}_{\kappa}^{ \pm}\right\rangle$are states of even or odd mirror parity constructed from the pair of HW functions as described in Sec. V C 1. These states are cell-periodic in plane and localized along $z$, and we also define new states $\left|\psi_{\kappa}^{ \pm}\right\rangle=e^{i \kappa \cdot \mathbf{r}}\left|\widetilde{h}_{\kappa}^{ \pm}\right\rangle$that are Wannier-like along $z$ and Bloch-like in plane.

When the Chern numbers $\widetilde{C}_{\mathrm{A}^{ \pm}}$are nonzero, it becomes impossible to choose a gauge for the states $\left|\psi_{\kappa}^{ \pm}\right\rangle$that is both smooth and periodic in the projected 2D BZ [5]. We assume a square $\mathrm{BZ}$ with $k_{x}, k_{y} \in[0,2 \pi]$, and choose a smooth but nonperiodic gauge for the $\left|\psi_{\kappa}^{-}\right\rangle$states. To characterize the lack of periodicity, let the phase relations between the edges of the BZ be

$$
\left|\psi_{\mathrm{R}}^{-}\right\rangle=e^{-i \mu}\left|\psi_{\mathrm{L}}^{-}\right\rangle, \quad\left|\psi_{\mathrm{T}}^{-}\right\rangle=e^{-i v}\left|\psi_{\mathrm{B}}^{-}\right\rangle,
$$

where $\{\mathrm{L}, \mathrm{R}, \mathrm{T}, \mathrm{B}\}=\{$ left,right,top,bottom $\}, \mu=\mu\left(k_{y}\right)$, and $v=v\left(k_{x}\right)$. Also let

$$
\Delta \mu=\mu(2 \pi)-\mu(0), \quad \Delta v=v(2 \pi)-v(0) .
$$

When computing the Berry phase around the BZ boundary as an integral of the connection $\mathbf{A}_{\kappa}^{-}=i\left\langle\widetilde{h}_{\kappa}^{-} \mid \partial_{\kappa} \widetilde{h}_{\kappa}^{-}\right\rangle$,

$$
\phi_{-}=\oint_{\partial \mathrm{BZ}} \mathbf{A}_{\kappa}^{-} \cdot d \boldsymbol{\kappa}
$$

the contribution from the $\mathrm{L}$ and $\mathrm{R}$ segments cancel except for terms coming from $\mu$, and similarly for the top and bottom segments. It follows that

$$
\phi_{-}=\Delta \mu-\Delta \nu
$$

We assume a smooth but nonperiodic gauge for the $\left|\psi_{\kappa}^{+}\right\rangle$ states as well, so that the phase $\gamma_{\kappa}$ in Eq. (B1) becomes a smooth function of $\kappa$ (except at the nodes, where $f_{\kappa}$ vanishes and $\gamma_{\kappa}$ becomes ill defined). Now we phase align $\left|\psi_{\kappa}^{+}\right\rangle$with $\left|\psi_{\kappa}^{-}\right\rangle$by regauging as follows,

$$
\left|\psi_{\kappa}^{+}\right\rangle^{\prime}=e^{i \gamma_{\kappa}}\left|\psi_{\kappa}^{+}\right\rangle
$$

(In this new gauge $f_{\kappa}^{\prime}$ is real, and $\gamma_{\kappa}^{\prime}$ is zero everywhere.) This will make a gauge for $\left|\psi_{\kappa}^{+}\right\rangle^{\prime}$ that is also nonperiodic. For the moment we assume only that this gauge is smooth in a neighborhood extending some small distance inside the boundary; we ignore what is going on deeper inside. It is not hard to see that the same relations as in Eq. (B2), with the same functions $\mu$ and $\nu$, apply to the $\left|\psi_{\kappa}^{+}\right\rangle^{\prime}$ states, and it follows that

$$
\phi_{+}^{\prime}=\phi_{-} \quad(\text { call it } \phi) .
$$

Now, in the case of the $\left|\psi_{\kappa}^{-}\right\rangle$states the interior was smooth, so by applying Stokes' theorem to

$$
2 \pi \widetilde{C}_{\mathrm{A}^{-}}=\int_{\mathrm{BZ}} \Omega_{\kappa}^{-} d^{2} k
$$

where $\Omega_{\kappa}^{-}=\partial_{k_{x}} A_{\kappa, y}^{-}-\partial_{k_{y}} A_{\kappa, x}^{-}$is the Berry curvature of state $\left|u_{\kappa}^{-}\right\rangle$, we get

$$
2 \pi \widetilde{C}_{\mathrm{A}^{-}}=\phi .
$$

If the interior of $\left|\psi_{\kappa}^{+}\right\rangle^{\prime}$ were also smooth, we would conclude that $\widetilde{C}_{\mathrm{A}^{+}}=\widetilde{C}_{\mathrm{A}^{-}}$. Conversely, when the MCN is nonzero there must exist nonanalytic points where the phase of $\left|u_{\kappa}^{+}\right\rangle^{\prime}$ changes discontinuously. Those points are precisely the nodes of $f_{\kappa}$, which we label by $j$; they act as vortex singularities of the Berry connection

$$
\left(\mathbf{A}_{\kappa}^{+}\right)^{\prime}=\mathbf{A}_{\kappa}^{+}-\partial_{\kappa} \gamma_{\kappa},
$$

and we extract their winding numbers $W_{j}$ using Eq. (28).

Let $S$ be the interior of the projected BZ with a small circle $c_{j}$ cut around each node, and apply Stokes' theorem over the region $S$ to find

$$
\int_{S} \Omega_{\boldsymbol{\kappa}}^{+} d^{2} k=\int_{\partial \mathrm{BZ}}\left(\mathbf{A}_{\kappa}^{+}\right)^{\prime} \cdot d \boldsymbol{\kappa}-\sum_{j} \oint_{c_{j}}\left(\mathbf{A}_{\boldsymbol{\kappa}}^{+}\right)^{\prime} \cdot d \boldsymbol{\kappa} .
$$

The first term on the right-hand side is equal to $\phi_{+}^{\prime}=\phi=$ $2 \pi \widetilde{C}_{\mathrm{A}^{-}}$. In the limit of small circles the left-hand side becomes $2 \pi \widetilde{C}_{\mathrm{A}^{+}}$, and the second term on the right-hand side reduces to $2 \pi \sum_{j} W_{j}$ [this follows from Eq. (B10) by noting that $\mathbf{A}_{\kappa}^{+}$is smooth everywhere]. Thus $\widetilde{C}_{\mathrm{A}^{+}}-\widetilde{C}_{\mathrm{A}^{-}}$equals $W_{\mathrm{A}}=$ $\sum_{j \in \mathrm{A}} W_{j}$, which is what we set out to prove. The same result holds if more than one pair of bands meet at some of the point nodes, in which case $\gamma_{\kappa}$ is given by the more general expression in Eq. (50).

\section{APPENDIX C: PHASE DIAGRAM OF THE MODIFIED DIRAC MODEL ON A CUBIC LATTICE}

In this Appendix, we map out the topological phase diagram of the model of Eq. (54) as a function of the parameters $m$ and $M$, for $c=1.0$. The band gap closes for $m=$ $0,4 M, 8 M, 12 M$ at the points $\Gamma, \mathrm{X}, \mathrm{M}$, and $\mathrm{A}$, respectively 

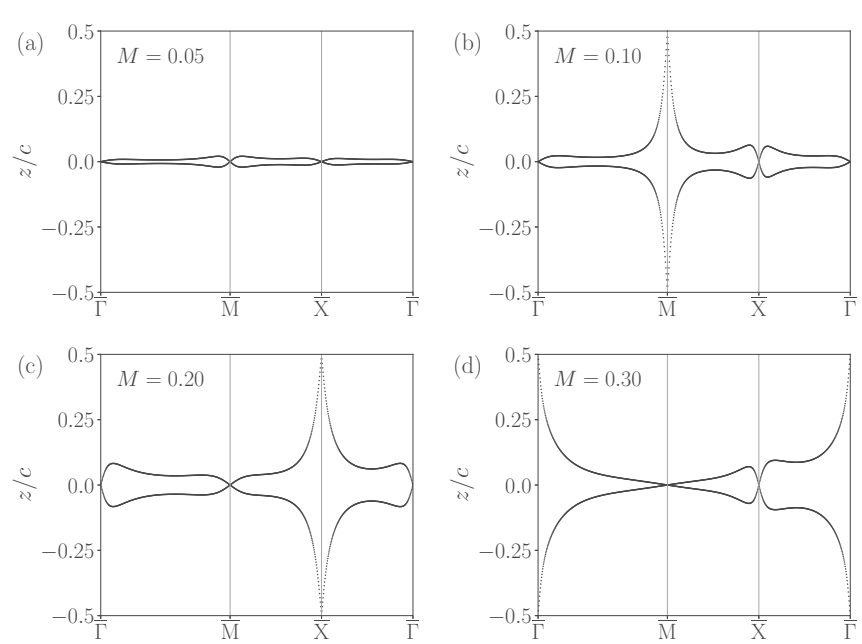

FIG. 10. Wannier bands of the modified Dirac model on a cubic lattice [Eq. (54)], for $m=1.0$ and varying $M$.

[43]. Those lines in the phase diagram mark the topological phase transitions between axion-even and axion-odd phases.
To decide which phases are trivial and which are topological, it is sufficient to inspect the Wannier band structures in Fig. 10, obtained for representative states in each of the four phases along the $m=1.0$ line. Since the model has several axion-odd symmetries (time reversal, inversion, and multiple mirrors), we can base our analysis on either of them, applying in each case the rules given in Ref. [13] to determine the axion $\mathbb{Z}_{2}$ index. In the following, we choose to focus on time-reversal symmetry.

The Wannier spectrum of an axion-odd phase with spinful time-reversal symmetry must be gapless, with each band touching the band above at one of the four TRIM and the band below at the other three (or vice versa). From this criterion we conclude that Figs. 10(a) and 10(c) correspond to axion-trivial phases, and Figs. 10(b) and 10(d) to axion-odd topological phases. Hence the system is topological for $0<m / M<4$ and $8<m / M<12$, producing the phase diagram in Fig. 6 . This is in agreement with Ref. [43], where the strong topological index $\nu_{0}=\theta / \pi$ of each phase was determined $a$ from the parity eigenvalues of the Bloch states at the eight TRIM in the 3D BZ [11].
[1] J. C. Y. Teo, L. Fu, and C. L. Kane, Surface states and topological invariants in three-dimensional topological insulators: Application to $\mathrm{Bi}_{1-x} \mathrm{Sb}_{x}$, Phys. Rev. B 78, 045426 (2008).

[2] Y. Ando and L. Fu, Topological crystalline insulators and topological superconductors: From concepts to materials, Annu. Rev. Condens. Matter Phys. 6, 361 (2015).

[3] X.-L. Qi, T. L. Hughes, and S.-C. Zhang, Topological field theory of time-reversal invariant insulators, Phys. Rev. B 78, 195424 (2008).

[4] A. M. Essin, J. E. Moore, and D. Vanderbilt, Magnetoelectric Polarizability and Axion Electrodynamics in Crystalline Insulators, Phys. Rev. Lett. 102, 146805 (2009).

[5] D. Vanderbilt, Berry Phases in Electronic Structure Theory: Electric Polarization, Orbital Magnetization and Topological Insulators (Cambridge University Press, Cambridge, 2018).

[6] N. P. Armitage and L. Wu, On the matter of topological insulators as magnetoelectrics, SciPost Phys. 6, 46 (2019).

[7] D. M. Nenno, C. A. C. Garcia, J. Gooth, C. Felser, and P. Narang, Axion physics in condensed-matter systems, Nat. Rev. Phys. 2, 682 (2020).

[8] A. Sekine and K. Nomura, Axion electrodynamics in topological materials, J. Appl. Phys. 129, 141101 (2021).

[9] M. M. Otrokov et al., Prediction and observation of an antiferromagnetic topological insulator, Nature (London) 576, 416 (2019).

[10] R. S. K. Mong, A. M. Essin, and J. E. Moore, Antiferromagnetic topological insulators, Phys. Rev. B 81, 245209 (2010).

[11] L. Fu and C. L. Kane, Topological insulators with inversion symmetry, Phys. Rev. B 76, 045302 (2007).

[12] A. M. Turner, Y. Zhang, R. S. K. Mong, and A. Vishwanath, Quantized response and topology of magnetic insulators with inversion symmetry, Phys. Rev. B 85, 165120 (2012).
[13] N. Varnava, I. Souza, and D. Vanderbilt, Axion coupling in the hybrid Wannier representation, Phys. Rev. B 101, 155130 (2020).

[14] D. Varjas, F. de Juan, and Y.-M. Lu, Bulk invariants and topological response in insulators and superconductors with nonsymmorphic symmetries, Phys. Rev. B 92, 195116 (2015).

[15] I. C. Fulga, N. Avraham, H. Beidenkopf, and A. Stern, Coupledlayer description of topological crystalline insulators, Phys. Rev. B 94, 125405 (2016).

[16] T. H. Hsieh, H. Lin, J. Liu, W. Duan, A. Bansil, and L. Fu, Topological crystalline insulators in the SnTe material class, Nat. Commun. 3, 982 (2012).

[17] J. Liu, T. H. Hsieh, P. Wei, W. Duan, J. Moodera, and L. Fu, Spin-filtered edge states with an electrically tunable gap in a two-dimensional topological crystalline insulator, Nat. Mater. 13, 178 (2014).

[18] N. Marzari and D. Vanderbilt, Maximally localized generalized Wannier functions for composite energy bands, Phys. Rev. B 56, 12847 (1997).

[19] M. Taherinejad and D. Vanderbilt, Adiabatic Pumping of Chern-Simons Axion Coupling, Phys. Rev. Lett. 114, 096401 (2015).

[20] B. Sutherland, Localization of electronic wave functions due to local topology, Phys. Rev. B 34, 5208 (1986).

[21] E. H. Lieb, Two Theorems on the Hubbard Model, Phys. Rev. Lett. 62, 1201 (1989).

[22] A. Ramachandran, A. Andreanov, and S. Flach, Chiral flat bands: Existence, engineering, and stability, Phys. Rev. B 96, 161104(R) (2017).

[23] J. A. Asbóth, A. Pályi, and L. Oroszlány, A Short Course on Topological Insulators (Springer, Cham, 2016).

[24] Y. Kim, C. L. Kane, E. J. Mele, and A. M. Rappe, Layered Topological Crystalline Insulators, Phys. Rev. Lett. 115, 086802 (2015). 
[25] B. J. Wieder and B. A. Bernevig, The axion insulator as a pump of fragile topology, arXiv:1810.02373.

[26] C.-H. Park and N. Marzari, Berry phase and pseudospin winding number in bilayer graphene, Phys. Rev. B 84, 205440 (2011).

[27] The PYтнTB code package is available at http://www.physics. rutgers.edu/pythtb/about.html.

[28] J. Enkovaara, C. Rostgaard, J. J. Mortensen, J. Chen, M. Dułak, L. Ferrighi, J. Gavnholt, C. Glinsvad, V. Haikola, H. A. Hansen et al., Electronic structure calculations with GPAW: A real-space implementation of the projector augmented-wave method, J. Phys.: Condens. Matter 22, 253202 (2010).

[29] A. A. Mostofi, J. R. Yates, G. Pizzi, Y.-S. Lee, I. Souza, D. Vanderbilt, and N. Marzari, An updated version of wannier90: A tool for obtaining maximally-localised Wannier functions, Comput. Phys. Commun. 185, 2309 (2014).

[30] T. Olsen, Designing in-plane heterostructures of quantum spin Hall insulators from first principles: $1 \mathrm{~T}^{\prime}-\mathrm{MoS}_{2}$ with adsorbates, Phys. Rev. B 94, 235106 (2016).

[31] J. P. Perdew, K. Burke, and M. Ernzerhof, Generalized Gradient Approximation Made Simple, Phys. Rev. Lett. 77, 3865 (1996).

[32] J. P. Perdew, K. Burke, and M. Ernzerhof, Generalized Gradient Approximation Made Simple [Phys. Rev. Lett. 77, 3865 (1996)], Phys. Rev. Lett. 78, 1396(E) (1997).

[33] P. E. Blöchl, Projector augmented-wave method, Phys. Rev. B 50, 17953 (1994).

[34] I. Souza, N. Marzari, and D. Vanderbilt, Maximally localized Wannier functions for entangled energy bands, Phys. Rev. B 65 , 035109 (2001).
[35] T. Olsen, E. Andersen, T. Okugawa, D. Torelli, T. Deilmann, and K. S. Thygesen, Discovering two-dimensional topological insulators from high-throughput computations, Phys. Rev. Mater. 3, 024005 (2019).

[36] S. Haastrup, M. Strange, M. Pandey, T. Deilmann, P. S. Schmidt, N. F. Hinsche, M. N. Gjerding, D. Torelli, P. M. Larsen, A. C. Riis-Jensen et al., The Computational 2D Materials Database: High-throughput modeling and discovery of atomically thin crystals, 2D Mater. 5, 042002 (2018).

[37] K. Kobayashi, Electronic states of SnTe and PbTe (001) monolayers with supports, Surf. Sci. 639, 54 (2015).

[38] J. Liu, X. Qian, and L. Fu, Crystal field effect induced topological crystalline insulators in monolayer IV-VI semiconductors, Nano Lett. 15, 2657 (2015).

[39] Y. Tanaka, Z. Ren, T. Sato, K. Nakayama, S. Souma, T. Takahashi, K. Segawa, and Y. Ando, Experimental realization of a topological crystalline insulator in SnTe, Nat. Phys. 8, 800 (2012).

[40] D. Gresch, G. Autès, O. V. Yazyev, M. Troyer, D. Vanderbilt, B. A. Bernevig, and A. A. Soluyanov, Z2Pack: Numerical implementation of hybrid Wannier centers for identifying topological materials, Phys. Rev. B 95, 075146 (2017).

[41] S.-Q. Shen, W.-Y. Shan, and H.-Z. Lu, Topological insulator and the Dirac equation, SPIN 01, 33 (2011).

[42] T. Rauch, H. Nguyen Minh, J. Henk, and I. Mertig, Model for ferromagnetic Weyl and nodal line semimetals: Topological invariants, surface states, anomalous and spin Hall effect, Phys. Rev. B 96, 235103 (2017).

[43] S.-Q. Shen, Topological Insulators-Dirac Equation in Condensed Matters (Springer, Berlin, 2012). 